\title{
Sobolev homeomorphisms with gradients of low rank via laminates
}

\author{
Daniel Faraco, Carlos Mora-Corral and Marcos Oliva \\ Department of Mathematics, Faculty of Sciences, Universidad Autónoma de Madrid, E-28049 Madrid, Spain \\ ICMAT CSIC-UAM-UCM-UC3M, E-28049 Madrid, Spain
}

July 14, 2021

\begin{abstract}
Let $\Omega \subset \mathbb{R}^{n}$ be a bounded open set. Given $2 \leq m \leq n$, we construct a convex function $\phi: \Omega \rightarrow \mathbb{R}$ whose gradient $f=\nabla \phi$ is a Hölder continuous homeomorphism, $f$ is the identity on $\partial \Omega$, the derivative $D f$ has rank $m-1$ a.e. in $\Omega$ and $D f$ is in the weak $L^{m}$ space $L^{m, w}$. The proof is based on convex integration and staircase laminates.
\end{abstract}

\section{Introduction}

Let $n \in \mathbb{N}$ and let $\Omega \subset \mathbb{R}^{n}$ be a bounded open set. This paper deals with homeomorphisms $f: \Omega \rightarrow \mathbb{R}^{n}$ in the Sobolev class whose derivative $D f$ has rank less than $m$ at a.e. point, for a given $m \leq n$.

In [20], Hencl proves that there exists a homeomorphism $f$ in $W^{1, p}\left((0,1)^{n},(0,1)^{n}\right), 1 \leq$ $p<n$, such that its Jacobian determinant $J_{f}$ equals zero a.e. Notice that condition $p<n$ is necessary, since if $f \in W^{1, n}$ and $J_{f} \geq 0$, then $f$ satisfies the Luzin $(\mathrm{N})$ condition and, consequently, the area formula holds. Therefore, any $f \in W^{1, n}$ with $J_{f}=0$ a.e. satisfies

$$
|f(\Omega)| \leq \int_{\Omega} J_{f}=0
$$

and, hence, $f$ cannot be a homeomorphism. In fact, in [21] it is proved that if a Sobolev map $f$ is such that

$$
\lim _{\varepsilon \rightarrow 0} \int_{\Omega}|D f|^{n-\varepsilon}=0
$$

then $f$ satisfies condition $(\mathrm{N})$, whereas the construction of [6] elaborates on that of [20] to show that there exists a homeomorphism $f \in W^{1,1}\left((0,1)^{n}, \mathbb{R}^{n}\right)$ such that $J_{f}=0$ almost everywhere and $D f$ is in the grand Lebesgue space $L^{n)}$, i.e.,

$$
\sup _{0<\varepsilon \leq n-1} \varepsilon \int_{(0,1)^{n}}|D f|^{n-\varepsilon}<\infty
$$


Obviously, such $f$ cannot satisfy (10).

The construction of Hencl [20, has been further developed in [13, 7] to construct biSobolev homeomorphisms $f$ with $J_{f}=0$ almost everywhere and with zero minors of $D f$ almost everywhere.

All those constructions were based on a careful explicit construction and a limit process to obtain a Cantor set where the Jacobian is supported.

In this work we explore a different way of obtaining such kind of pathological maps by using the staircase laminates invented by Faraco [15], in combination with the version of convex integration used by Müller and Šverák [28]. In fact, such laminates have turned out to be useful in a number of apparently unrelated problems such as $L^{p}$ theory of elliptic equations [2], Burkholder functions [4, Hessians of rank-one convex functions [9], microstructure and phase transitions in solids [29, 27] and counterexamples of $L^{1}$ estimates [8]. As in the case of Ornstein inequalities [8], laminates allow one to decouple the construction of pathological maps occurring in various situations into an analytical part and a geometrical part. The analytical part is taken care by the general theory of laminates and the version of convex integration based on in-approximations (in fact, in the problem at hand, on a slight evolution of the version for unbounded sets developed in [2] which guarantees that the limit map is a homeomorphism). The geometrical part, which is the key in the whole process, consists in finding a suitable staircase laminate. In fact, in [16] it was sketched how to use laminates to obtain, in dimension 2, a convex function whose gradient $f$ is in $W^{1, p}$ for all $p<2$, and satisfies $J_{f}=0$ a.e., recovering another interesting example of Alberti and Ambrosio [1].

We mention that gradients of convex functions are interesting in its own right; for example, they are the key ingredient in the polar decomposition of the Brenier map in mass transportation [5]. In this regard, our example show some limits of the regularity for transport maps [1].

In the current work we show that with staircase laminates it is possible to combine the results of [16, 1, 20]. In fact, we also recover the result of Černý [7, where he constructs a homeomorphism $f$ with all its minors of $m$-th order equal to zero almost everywhere belonging to $W^{1, p}$ with $1 \leq p<\frac{n}{n+1-m}$.

To be precise, we build a probability measure formed by staircase laminates in the planes parallel to the axes, which can be pushed to show that not only the Jacobian is zero but also that $D f$ has all its minors of order $m$ equal to zero almost everywhere and $D f$ is in $L^{m, w}$, i.e., there exists a constant $C>0$ such that

$$
|\{x \in \Omega:|D f(x)|>t\}| \leq C t^{-m}, \quad t>0 .
$$

In the particular case when $m=n$ we have $L^{n, w} \subset L^{n)}$, so our result is sharp in the sense explained before. In fact, it seems likely that we could push our construction so that $D f(x)$ is diagonal for all $x$ except in a set of arbitrarily small measure, but we do not pursue this issue here.

This is the theorem that we will prove.

Theorem 1. Let $\Omega \subset \mathbb{R}^{n}$ be a bounded open set, $m \in \mathbb{N}, 2 \leq m \leq n, \delta>0$ and $\alpha \in(0,1)$. Then there exists a convex function $u: \Omega \rightarrow \mathbb{R}$ such that its gradient $f=\nabla u$ satisfies: 
i) $f \in W^{1,1}\left(\Omega, \mathbb{R}^{n}\right)$ and $f: \Omega \rightarrow \Omega$ is a homeomorphism.

ii) $f=\mathrm{id}$ on $\partial \Omega$.

iii) $\operatorname{rank}(D f(x))<m$ for a.e. $x \in \Omega$.

iv) $D f \in L^{m, w}\left(\Omega, \mathbb{R}^{n \times n}\right)$.

v) $\|f-\mathrm{id}\|_{C^{\alpha}(\bar{\Omega})}<\delta$ and $\left\|f^{-1}-\mathrm{id}\right\|_{C^{\alpha}(\bar{\Omega})}<\delta$.

Our theorem shows that a Hölder continuous Brenier map (the gradient of a convex function; see, e.g., [30, p. 67] for the definition) may have a rather pathological behaviour.

As discussed at the beginning, the result is known to be sharp in the sense of integrability in the case $m=n$. Notice that in this case, as explained in [20], using the area formula for Sobolev mappings ([19]) we have that this kind of homeomorphisms sends a set of full measure to a null set, and a null set to a set of full measure, i.e., there exists $Z \subset \Omega$ of measure zero such that

$$
|f(\Omega \backslash Z)|=\int_{f(\Omega \backslash Z)} d y=\int_{\Omega \backslash Z} J_{f}(x) d x=0
$$

and

$$
|f(Z)|=|f(\Omega)|-|f(\Omega \backslash Z)|=|f(\Omega)| .
$$

The structure of the paper is as follows.

In Section 2 we introduce the concept of laminate of finite order and sketch the construction of the laminate that will be central in the proof of Theorem 1, Notice that if $m \neq n \neq 2$ the construction is genuinely different from previous staircase laminates as we need to lower the rank accordingly.

Section 3 presents the general notation of the paper.

In Section 4 we prove that, given a laminate of finite order, there exists a function $f$ whose derivative is close to the laminate. Moreover, if the laminate is supported in the set of positive definite matrices then $f$ is a homeomorphism.

Section 5 shows that if we modify a Hölder homeomorphism by cutting and pasting, the map obtained is still a Hölder homeomorphism .

Section 6, which is the bulk of the paper, constructs a sequence of laminates that converges to the probability measure sketched in Section 2, as well as a sequence of functions that approximate the laminates.

In Section 7 we prove Theorem 1 .

In Section 8 we show the sharpness of our result by proving that there does not exist a Hölder continuous homeomorphism in $W^{1, m}\left(\Omega, \mathbb{R}^{n}\right)$ such that $f=$ id on $\partial \Omega$ and $\operatorname{rank}(D f)<$ $m$ a.e. in $\Omega$. Moreover, Hölder continuity can be dispensed with if $f \in W^{1, p}\left(\Omega, \mathbb{R}^{n}\right)$ for somw $p>m$.

Note: When our paper was presented, S. Hencl called our attention to a recent preprint by Liu and Malý [25, where a result very similar to our Theorem 1 was proved, with a construction related to laminates but not inspired in [16]. It would be very interesting to see how much these examples have in common. For instance, an understanding of the support of 
the distributional Jacobian or, in general, the distributional minors is pending. The advantage of the method presented in this paper is that, once an extremal staircase laminate is found, which is a relatively fast task (Section 2), the extremal mapping quite likely will appear, for example by the in-approximation method. Notice that this last step is not always possible, as shown by the case of monotone maps (the staircase laminate from [8] is supported in the range of gradients of planar monotone maps, which are regular by [1]). Finally, we mention that it will also be worthy to see the relation with the works [22, 23, where the results of [8] are easily recovered from the study of rank-one convex functions that are one-homogeneous.

\section{Sketch of the proof}

The next definition introduces the concept of laminate of finite order [10, 29, 28, 2].

Definition 2. The family $\mathcal{L}\left(\mathbb{R}^{n \times n}\right)$ of laminates of finite order is the smallest family of probability measures in $\mathbb{R}^{n \times n}$ with the properties:

- $\mathcal{L}\left(\mathbb{R}^{n \times n}\right)$ contains all the Dirac masses.

- If $\sum_{i=1}^{N} \lambda_{i} \delta_{A_{i}} \in \mathcal{L}\left(\mathbb{R}^{n \times n}\right)$ and $A_{N}=\lambda B+(1-\lambda) C$, where $\lambda \in[0,1]$ and $\operatorname{rank}(B-C)=1$, then the probability measure

$$
\sum_{i=1}^{N-1} \lambda_{i} \delta_{A_{i}}+\lambda_{N}\left(\lambda \delta_{B}+(1-\lambda) \delta_{C}\right)
$$

is also in $\mathcal{L}\left(\mathbb{R}^{n \times n}\right)$.

Note that any laminate of finite order is a convex combination of Dirac masses. Since in this work we will only use laminates of finite order, for simplicity they will be just called laminates.

In this section we construct the sequence of laminates $\nu_{k}$ of finite order that is behind the whole article. The actual proof will consist in approximating $\nu_{k}$ with laminates of finite order supported in the set of positive definite matrices, then use Proposition 3 to obtain homeomorphisms that are close to the approximate laminates in small regions of the domain, then paste the obtained homeomorphisms to construct a homeomorphism in the whole domain and, finally, a limit passage will yield the homeomorphism $f$ of Theorem 1 . The fact that $f$ is the gradient of a convex function is standard since $D f$ was constructed to be symmetric positive semidefinite.

Although this section is not necessary for the proof of Theorem 1, it will help the reader to follow the construction of Section 6 ,

In order to construct $\nu_{k}$, we need to define the sets

$$
S_{i}^{k}=\left\{A=k \operatorname{diag}(v): v \in\{0,1\}^{n} \text { and } \operatorname{rank}(A)=n-i\right\}, \quad k \in \mathbb{N}, \quad i \in\{0, \ldots, n-m\}
$$

and

$$
E=\left\{A \in \mathbb{R}^{n \times n}: \operatorname{rank}(A)<m\right\}
$$


Thus, the matrices of $S_{i}^{k}$ are $k$ times the identity matrix in the $(n-i)$-dimensional linear subspaces parallel to the axes.

The main property of the laminates to be constructed is as follows: for each $k \in \mathbb{N}, \nu_{k}$ is supported in $\bigcup_{i=0}^{n-m} S_{i}^{k} \cup E$,

$$
|A| \leq k \text { for all } A \in \operatorname{supp}\left(\nu_{k}\right)
$$

and

$$
\nu_{k}\left(S_{i}^{k}\right) \leq C k^{i-n}
$$

for some $C>0$.

The weak ${ }^{*}$ limit $\nu$ of $\nu_{k}$ is supported in the set $E$. It satisfies that there exists a constant $C>0$ such that

$$
\nu(\{|A|>t\}) \leq C t^{-m}, \quad t>0
$$

This last inequality will give us the desired integrability of the derivative of the homeomorphism.

The laminates $\nu_{k}$ are defined inductively as follows. We start with $\nu_{1}=\delta_{I}$. Now, given

$$
\nu_{k-1}=\sum_{j=1}^{N} \lambda_{j} \delta_{A_{j}} \in \mathcal{L}\left(\mathbb{R}^{n \times n}\right),
$$

with $A_{j} \in \bigcup_{i=0}^{n-m} S_{i}^{k-1} \cup E$, all different, we are going to split the matrices of $S_{i}^{k-1}$ in matrices in $\bigcup_{i=0}^{n-m} S_{i}^{k} \cup E$ following rank-one lines as in Definition 2 ,

Let $A \in \operatorname{supp}\left(\nu_{k-1}\right)$. If $A \in E$ we define $\nu_{A}=\delta_{A}$, whereas if $A \in S_{i}^{k-1}$ for some $i \in\{0, \ldots, n-m\}$, we construct $\nu_{A}$ inductively. Without loss of generality,

$$
A=(k-1) \operatorname{diag}(\underbrace{1, \ldots, 1}_{n-i}, \underbrace{0, \ldots, 0}_{i}) .
$$

We shall construct families

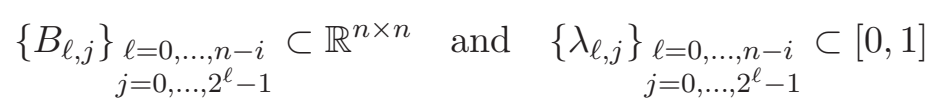

by finite induction on $\ell$.

Let $B_{0,0}=A, \lambda_{0,0}=1$ and for $0 \leq \ell \leq n-i-1,0 \leq j \leq 2^{\ell}-1$, we assume that $\left\{B_{\ell, j}\right\}_{j=0}^{2^{\ell}-1}$ and $\left\{\lambda_{\ell, j}\right\}_{j=0}^{2^{\ell}-1}$ have been defined, $B_{\ell, j}$ are diagonal, $\lambda_{\ell, j} \geq 0$,

$$
\begin{gathered}
\sum_{j=0}^{2^{\ell}-1} \lambda_{\ell, j}=1, \quad B_{0,0}=\sum_{j=0}^{2^{\ell}-1} \lambda_{\ell, j} B_{\ell, j} \\
\sum_{j=0}^{2^{\ell}-1} \lambda_{\ell, j} \delta_{B_{\ell, j}} \in \mathcal{L}\left(\mathbb{R}^{n \times n}\right)
\end{gathered}
$$


and

$$
\left(B_{\ell, j}\right)_{\alpha, \alpha}= \begin{cases}k-1 & \text { if } \alpha=\ell+1, \ldots, n-i, \\ 0 & \text { if } \alpha=n-i+1, \ldots, n .\end{cases}
$$

We also assume that if $B_{\ell, j} \notin E$ then

$$
\left(B_{\ell, j}\right)_{\alpha, \alpha} \in\{0, k\}, \quad \alpha=1, \ldots, \ell
$$

$$
\lambda_{\ell, j}=\frac{(k-1)^{\beta_{\ell, j}}}{k^{\ell}}
$$

where

$$
\beta_{\ell, j}:=\#\left\{\alpha \in\{1, \ldots, \ell\}:\left(B_{\ell, j}\right)_{\alpha, \alpha}=k\right\}=\operatorname{rank}\left(B_{\ell, j}\right)-n+i+\ell,
$$

and for each $B_{\ell, j^{\prime}} \notin E$ with $j^{\prime} \neq j$, we have $B_{\ell, j^{\prime}} \neq B_{\ell, j}$.

With the above induction hypotheses, we construct $\left\{B_{\ell+1, j}\right\}_{j=0}^{2^{\ell+1}-1}$ and $\left\{\lambda_{\ell+1, j}\right\}_{j=0}^{2^{\ell+1}-1}$ as follows. For any $0 \leq j \leq 2^{\ell}-1$, we define

$$
\begin{gathered}
B_{\ell+1,2 j}= \begin{cases}B_{\ell, j}-\operatorname{diag}(\underbrace{0, \ldots, 0}_{\ell}, k-1, \underbrace{0, \ldots, 0}_{n-\ell-1}), & \text { if } B_{\ell, j} \notin E, \\
B_{\ell, j}, & \text { if } B_{\ell, j} \in E,\end{cases} \\
B_{\ell+1,2 j+1}= \begin{cases}B_{\ell, j}+\operatorname{diag}(\underbrace{0, \ldots, 0}_{\ell}, 1 \underbrace{0, \ldots, 0}_{n-\ell-1}), & \text { if } B_{\ell, j} \notin E, \\
B_{\ell, j}, & \text { if } B_{\ell, j} \in E,\end{cases} \\
\lambda_{\ell+1,2 j}=\lambda_{\ell, j} \frac{1}{k} \quad \text { and } \quad \lambda_{\ell+1,2 j+1}=\lambda_{\ell, j} \frac{k-1}{k} .
\end{gathered}
$$

Now we check the induction hypotheses.

We have $B_{\ell, j}=\frac{1}{k} B_{\ell+1,2 j}+\frac{k-1}{k} B_{\ell+1,2 j+1}$ and $\operatorname{rank}\left(B_{\ell+1,2 j}-B_{\ell+1,2 j+1}\right) \leq 1$, so property (6) holds for $\ell+1$. Analogously, equalities (5) are easily seen to hold for $\ell+1$ as well.

Now, let $0 \leq j \leq 2^{\ell+1}-1$. We have

$$
\left(B_{\ell+1, j}\right)_{\alpha, \alpha}=\left(B_{\ell,\left\lfloor\frac{j}{2}\right\rfloor}\right)_{\alpha, \alpha}, \quad \alpha \neq \ell+1
$$

and

$$
\left(B_{\ell+1, j}\right)_{\ell+1, \ell+1}= \begin{cases}0, & \text { if } B_{\ell,\left\lfloor\frac{j}{2}\right\rfloor} \notin E, j \text { even, } \\ k, & \text { if } B_{\ell,\left\lfloor\frac{j}{2}\right\rfloor} \notin E, j \text { odd } \\ \left(B_{\ell,\left\lfloor\frac{j}{2}\right\rfloor}\right)_{\ell+1, \ell+1}, & \text { if } B_{\ell,\left\lfloor\frac{j}{2}\right\rfloor} \in E .\end{cases}
$$


Therefore, equality (7) holds for $\ell+1$.

Now fix $\ell, j$ such that $B_{\ell+1, j} \notin E$. Then $B_{\ell,\left\lfloor\frac{j}{2}\right\rfloor} \notin E$ and, hence, property (8) holds for $\ell+1$. We also have

$$
\beta_{\ell+1, j}= \begin{cases}\beta_{\ell, \frac{j}{2}}, & j \text { even } \\ \beta_{\ell, \frac{j-1}{2}}+1, & j \text { odd }\end{cases}
$$

so (9) holds for $\ell+1$. Finally, let $j^{\prime} \neq j$ be such that $B_{\ell+1, j^{\prime}} \notin E$. If $\left\lfloor\frac{j}{2}\right\rfloor \neq\left\lfloor\frac{j^{\prime}}{2}\right\rfloor$, then $B_{\ell,\left\lfloor\frac{j^{\prime}}{2}\right\rfloor} \neq B_{\ell,\left\lfloor\frac{j}{2}\right\rfloor}$, and, hence, $B_{\ell+1, j^{\prime}} \neq B_{\ell+1, j}$, whereas if $\left\lfloor\frac{j}{2}\right\rfloor=\left\lfloor\frac{j^{\prime}}{2}\right\rfloor$, then $\left(B_{\ell+1, j^{\prime}}\right)_{\ell+1, \ell+1} \neq$ $\left(B_{\ell+1, j}\right)_{\ell+1, \ell+1}$, and, hence, $B_{\ell+1, j^{\prime}} \neq B_{\ell+1, j}$. Here ends the inductive construction of the families (44) with the required properties.

Thanks to (7) and (8) we have, for all $0 \leq j \leq 2^{n-i}-1$,

$$
B_{n-i, j} \in \bigcup_{\ell=i}^{n-m} S_{\ell}^{k} \cup E
$$

whereas (9) yields

$$
\lambda_{n-i, j}=\frac{(k-1)^{\operatorname{rank}\left(B_{n-i, j}\right)}}{k^{n-i}} .
$$

We define

$$
\nu_{A}=\sum_{j=0}^{2^{n-i}-1} \lambda_{n-i, j} \delta_{B_{n-i, j}}
$$

which is a laminate due to (6).

From (10) we get

$$
\nu_{A}\left(\bigcup_{\ell=0}^{i-1} S_{\ell}^{k}\right)=0
$$

whereas for $\ell \in\{i, \ldots, n-m\}$, we have, due to (77) and (11)

$$
\nu_{A}\left(S_{\ell}^{k}\right)=\sum_{j: B_{n-i, j} \in S_{\ell}^{k}} \lambda_{n-i, j}=\sum_{j: B_{n-i, j} \in S_{\ell}^{k}} \frac{(k-1)^{n-\ell}}{k^{n-i}} \leq\left(\begin{array}{c}
n-i \\
n-\ell
\end{array}\right) \frac{(k-1)^{n-\ell}}{k^{n-i}}
$$

since the $B_{n-i, j}\left(0 \leq j \leq 2^{n-i}-1\right)$ not in $E$ are all different. Thus, for each $j \in\{1, \ldots, N\}$, given $A_{j}$ appearing in (3), we have constructed $\nu_{A_{j}}$ as in (12) if $A_{j} \notin E$ and $\nu_{A_{j}}=\delta_{A_{j}}$ if $A_{j} \in E$. So

$$
\nu_{A_{j}}\left(S_{\ell}^{k}\right)=0 \quad \text { if } A_{j} \in E, \quad \forall \ell \in\{0, \ldots, n-m\}
$$


and we define the probability measure $\nu_{k}:=\sum_{j=1}^{N} \lambda_{j} \nu_{A_{j}}$, which is supported in $\bigcup_{i=0}^{n-m} S_{i}^{k} \cup E$ by (10). In fact, $\nu_{k}$ is a laminate, but this is not important on the proof. We observe that we have proved

$$
|A| \leq k \text { for all } A \in \operatorname{supp}\left(\nu_{k}\right) \text {. }
$$

We also let

$$
C_{k}=\prod_{j=1}^{k-1}\left(1+2^{n} j^{-2}\right)
$$

and we will prove that for all $k \in \mathbb{N}$ and $i=0, \ldots, n-m$,

$$
\nu_{k}\left(S_{i}^{k}\right) \leq C_{k} k^{i-n} .
$$

We proceed by induction. The inequality for $k=1$ is immediate since $\nu_{1}=\delta_{I}$. Let $k \geq 2$ and suppose that for $i=0, \ldots, n-m$, inequality (18) holds for $k-1$. Since the $A_{j}$ of (3) are different, we have that $\nu_{k-1}\left(A_{j}\right)=\lambda_{j}$. Now, for all $\ell \in\{0, \ldots, n-m\}$, we use (13) and (15) to get

$$
\begin{aligned}
\nu_{k}\left(S_{\ell}^{k}\right) & =\sum_{j=1}^{N} \nu_{k-1}\left(A_{j}\right) \nu_{A_{j}}\left(S_{\ell}^{k}\right)=\left[\sum_{j: A_{j} \in E}+\sum_{i=0}^{n-m} \sum_{j: A_{j} \in S_{i}^{k-1}}\right] \nu_{k-1}\left(A_{j}\right) \nu_{A_{j}}\left(S_{\ell}^{k}\right) \\
& =\sum_{i=0}^{\ell} \sum_{j: A_{j} \in S_{i}^{k-1}} \nu_{k-1}\left(A_{j}\right) \nu_{A_{j}}\left(S_{\ell}^{k}\right) .
\end{aligned}
$$

We use that (18) is valid for $k-1$ to get that for all $i \in\{0, \ldots, n-m\}$,

$$
\frac{(k-1)^{n-\ell}}{k^{n-i}} \sum_{j: A_{j} \in S_{i}^{k-1}} \nu_{k-1}\left(A_{j}\right)=\frac{(k-1)^{n-\ell}}{k^{n-i}} \nu_{k-1}\left(S_{i}^{k-1}\right) \leq C_{k-1} \frac{(k-1)^{i-\ell}}{k^{n-i}} .
$$

In addition,

$$
\sum_{i=0}^{\ell}\left(\begin{array}{l}
n-i \\
n-\ell
\end{array}\right)((k-1) k)^{i-\ell} \leq 1+\sum_{i=0}^{\ell-1}\left(\begin{array}{l}
n-i \\
n-\ell
\end{array}\right)(k-1)^{2(i-\ell)} \leq 1+2^{n}(k-1)^{-2},
$$

where we have used the crude inequality $\sum_{i=0}^{\ell-1}\left(\begin{array}{c}n-i \\ n-\ell\end{array}\right) \leq 2^{n}$. We combine the last three inequalities and (14) to get

$$
\begin{aligned}
\nu_{k}\left(S_{\ell}^{k}\right) & =\sum_{i=0}^{\ell} \sum_{j: A_{j} \in S_{i}^{k-1}} \nu_{k-1}\left(A_{j}\right) \nu_{A_{j}}\left(S_{\ell}^{k}\right) \leq \sum_{i=0}^{\ell} \sum_{j: A_{j} \in S_{i}^{k-1}} \nu_{k-1}\left(A_{j}\right)\left(\begin{array}{c}
n-i \\
n-\ell
\end{array}\right) \frac{(k-1)^{n-\ell}}{k^{n-i}} \\
& \leq \sum_{i=0}^{\ell}\left(\begin{array}{c}
n-i \\
n-\ell
\end{array}\right) C_{k-1} \frac{(k-1)^{i-\ell}}{k^{n-i}} \leq k^{\ell-n} C_{k-1}\left(1+2^{n}(k-1)^{-2}\right)=C_{k} k^{\ell-n},
\end{aligned}
$$


which proves (18). We observe from (17) that $\lim _{k \rightarrow \infty} C_{k}<\infty$. Consequently,

$$
\nu_{k}\left(\bigcup_{i=0}^{n-m} S_{i}^{k}\right) \leq \sum_{i=0}^{n-m} C_{k} k^{i-n} \leq C k^{-m}
$$

for some $C>0$.

Next, we will prove by induction that exists $\tilde{C}>0$ such that for all $k \in \mathbb{N}$,

$$
\nu_{k}\left(\left\{A \in \mathbb{R}^{n \times n}:|A|>t\right\}\right) \leq \tilde{C} t^{-m}, \quad t>0 .
$$

For simplicity of notation, the set $\left\{A \in \mathbb{R}^{n \times n}:|A|>t\right\}$ will be abbreviated as $\{|A|>t\}$. When $k=1$, we have $\nu_{1}=\delta_{I}$, hence, (20) is obvious. Now, we divide the inductive step in three cases, according to the values of $t$.

If $t \geq k+1$, we use (16) to obtain that $\nu_{k+1}(\{|A|>t\})=0$.

In the case $t<k$, we first show that if $|A|>t, A_{j} \in \operatorname{supp}\left(\nu_{k}\right)$ and $\nu_{A_{j}}(A)>0$, then $\left|A_{j}\right|>t$. Indeed, if $A_{j} \in E$, then $\nu_{A_{j}}=\delta_{A_{j}}$, so, as $\nu_{A_{j}}(A)>0$, we have $A=A_{j}$, and therefore $\left|A_{j}\right|>t$; whereas if $A_{j} \in \bigcup_{i=0}^{n-m} S_{i}^{k}$, then $\left|A_{j}\right|=k>t$. Therefore, if $\left|A_{j}\right| \leq t$, then $\nu_{A_{j}}(\{|A|>t\})=0$, and, hence,

$$
\begin{aligned}
\nu_{k+1}(\{|A|>t\}) & =\sum_{\substack{j: A_{j} \in \operatorname{supp}\left(\nu_{k}\right) \\
\left|A_{j}\right|>t}} \nu_{k}\left(A_{j}\right) \nu_{A_{j}}(\{|A|>t\}) \\
& \leq \nu_{k}\left(\left\{A_{j} \in \operatorname{supp}\left(\nu_{k}\right):\left|A_{j}\right|>t\right\}\right) \leq \nu_{k}(\{|A|>t\}) \leq \tilde{C} t^{-m} .
\end{aligned}
$$

Finally, in the case $k \leq t<k+1$, we use that if $A \in \operatorname{supp}\left(\nu_{k+1}\right)$ and $|A|>t$, then, by (16), we have that for all $A_{j} \in \operatorname{supp}\left(\nu_{k}\right) \cap E$, equalities $\nu_{A_{j}}(A)=\delta_{A_{j}}(A)=0$ are satisfied. Hence, thanks to (19),

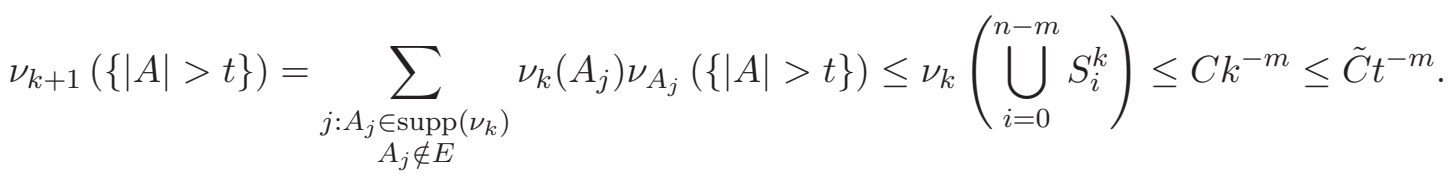

This finishes the proof of (20).

Let $\nu$ be the weak* limit of $\nu_{k}$ as $k \rightarrow \infty$. Thanks to (19), $\nu$ is supported in $E$ and, by (20), inequality (2) holds.

We will try to illustrate this construction with some pictures.

In the simplest case $n=m=2, E$ is the set of matrices with zero determinant, $\nu_{1}=\delta_{I}$ and the first steps of the construction are depicted in Figures 1 and 2, In the first step (Figure 11) we split $B_{0,0}=I$ into $B_{1,0}=\operatorname{diag}(0,1)$ and $B_{1,1}=\operatorname{diag}(2,1)$. As $B_{1,0} \in E$, the second step (Figure 2) consists in splitting $B_{1,1}$ into $B_{2,2}=\operatorname{diag}(2,0)$ and $B_{2,3}=\operatorname{diag}(2,2)$. After the second split, we obtain

$$
\nu_{2}=\nu_{I}=\sum_{j=0}^{3} \lambda_{2, j} \delta_{B_{2, j}}=\frac{1}{2} \delta_{\operatorname{diag}(0,1)}+\frac{1}{4} \delta_{\operatorname{diag}(2,0)}+\frac{1}{4} \delta_{\operatorname{diag}(2,2)} .
$$




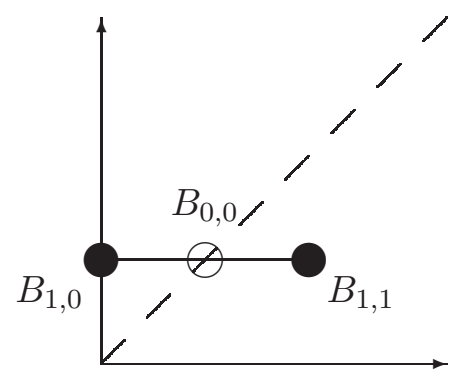

Figure 1: First split, $n=m=2, B_{0,0}=I: B_{1,0}=\operatorname{diag}(0,1), B_{1,1}=\operatorname{diag}(2,1)$.

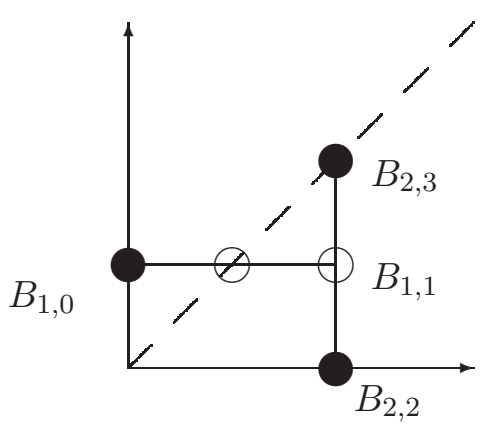

Figure 2: Second split, $n=m=2: B_{2,0}=B_{2,1}=B_{1,0}=\operatorname{diag}(0,1), B_{2,2}=\operatorname{diag}(2,0)$, $B_{2,3}=\operatorname{diag}(2,2)$.

In the case $n=3, m=2, E$ is the set of matrices of rank less than 2. In order to exemplify the passage from step $k-1$ to step $k$, if we start with a matrix in $S_{1}^{k-1}$, the construction is the same as in the case $n=m=2$, whereas if we start with $A \in S_{0}^{k-1}$, we have $A=(k-1) I$ and Figures 3, 4 and 5 show the construction of $\nu_{A}$.

We get at the end

$$
\begin{aligned}
\nu_{A} & =\sum_{j=0}^{7} \lambda_{3, j} \delta_{B_{3, j}}=\frac{1}{k^{2}} \delta_{\operatorname{diag}(0,0, k-1)}+\frac{(k-1)^{2}}{k^{3}} \delta_{\operatorname{diag}(k, 0, k)}+\frac{k-1}{k^{3}} \delta_{\operatorname{diag}(k, 0,0)} \\
& +\frac{(k-1)^{2}}{k^{3}} \delta_{\operatorname{diag}(0, k, k)}+\frac{k-1}{k^{3}} \delta_{\operatorname{diag}(0, k, 0)}+\frac{(k-1)^{2}}{k^{3}} \delta_{\operatorname{diag}(k, k, 0)}+\frac{(k-1)^{3}}{k^{3}} \delta_{k I} .
\end{aligned}
$$

The construction of $\nu_{k}$ would entail the analogous construction for each $A \in S_{0}^{k-1} \cup S_{1}^{k-1}$. 


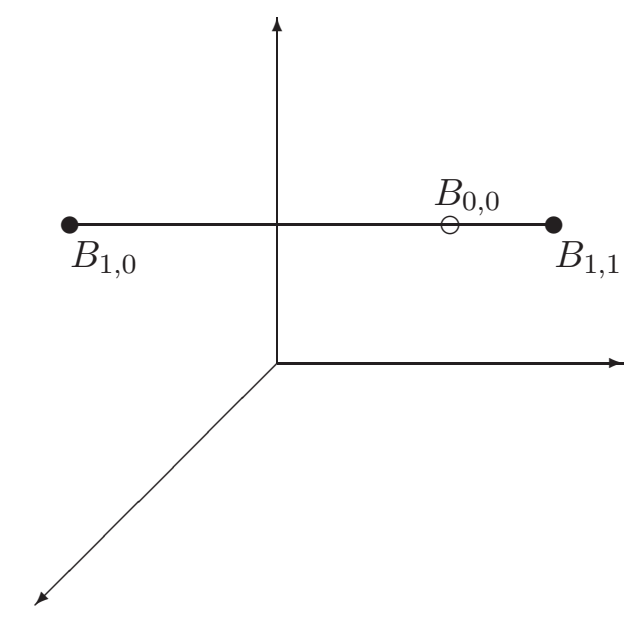

Figure 3: First split, $n=3, m=2: B_{0,0}=(k-1) I, B_{1,0}=\operatorname{diag}(k-1,0, k-1), B_{1,1}=$ $\operatorname{diag}(k-1, k, k-1)$.

\section{General notation}

We explain the general notation used throughout the paper, most of which is standard.

In the whole paper, $\Omega$ is an open, non-empty bounded set of $\mathbb{R}^{n}$.

We denote by $\mathbb{R}^{n \times n}$ the set of $n \times n$ matrices, by $\Gamma_{+}$its subset of symmetric positive semidefinite matrices, and by $S O(n) \subset \mathbb{R}^{n \times n}$ the orthogonal matrices with determinant 1 .

Given $A_{i} \in \mathbb{R}^{n \times n}$, the measure $\delta_{A_{i}}$ is the Dirac delta at $A_{i}$. The barycenter of the probability measure $\nu=\sum_{i=1}^{N} \alpha_{i} \delta_{A_{i}}$ is $\bar{\nu}=\sum_{i=1}^{N} \alpha_{i} A_{i}$.

Given $A \in \mathbb{R}^{n \times n}$, let $\sigma_{1}(A) \leq \cdots \leq \sigma_{n}(A)$ denote its singular values. If the matrix $A$ is clear from the context, we will just indicate their singular values as $\sigma_{1}, \ldots, \sigma_{n}$. In fact, in this paper we will always deal with $A \in \Gamma_{+}$, so its eigenvalues coincide with its singular values. Its components are written $A_{\alpha, \beta}$ for $\alpha, \beta \in\{1, \ldots, n\}$. Its operator norm is denoted by $|A|$, which coincides with $\sigma_{n}(A)$. The norm of a $v \in \mathbb{R}^{n}$ is also denoted by $|v|$.

Given $a_{1}, \ldots, a_{n} \in \mathbb{R}$ the matrix $\operatorname{diag}\left(a_{1}, \ldots, a_{n}\right) \in \mathbb{R}^{n \times n}$ is the diagonal matrix with diagonal entries $a_{1}, \ldots, a_{n}$.

We will use the symbol $\lesssim$ when there exists a constant depending only on $n$ and $m$ such that the left hand side is less than or equal to the constant times the right hand side.

Given a set $E \subset \mathbb{R}^{n}$, we denote its characteristic function by $\chi_{E}$. We write $\# E$ for the number of elements of $E$. Its Lebesgue measure is denoted by $|E|$.

Given $a \in \mathbb{R}$, its integer part is denoted by $\lfloor a\rfloor$.

Given $E \subset \mathbb{R}^{n}, \alpha \in(0,1]$ and a function $f: E \rightarrow \mathbb{R}^{n}$, we denote the Hölder seminorm, 


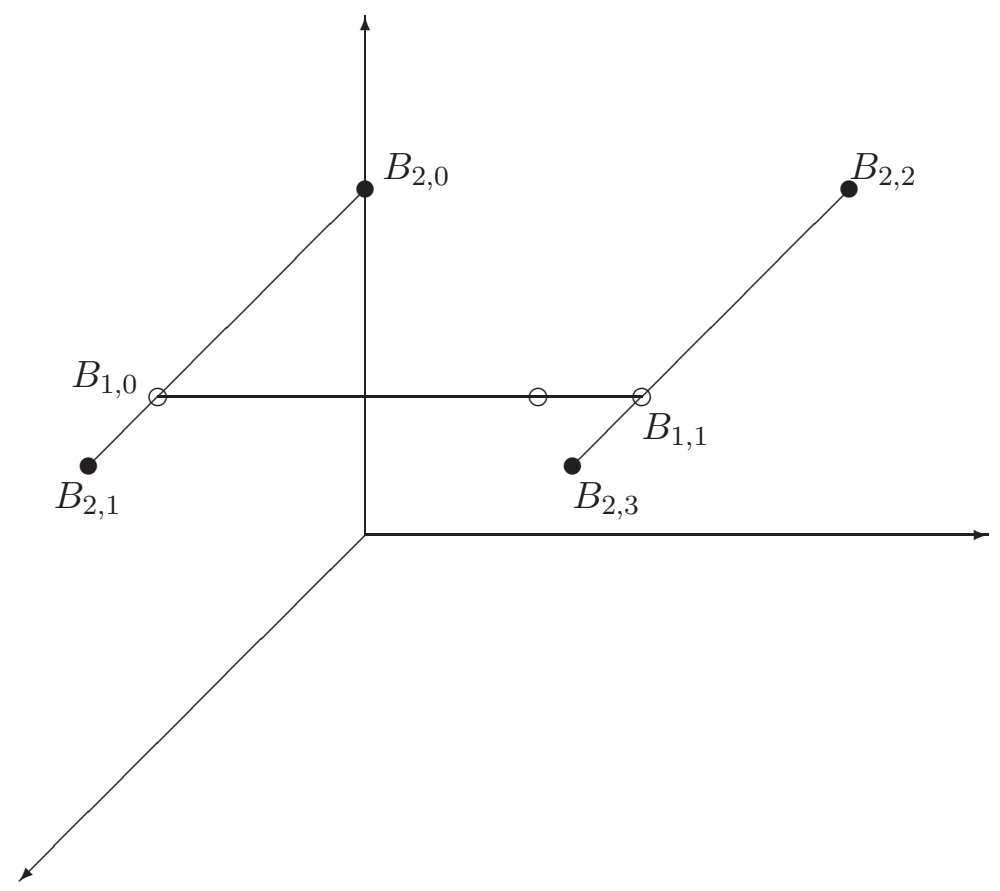

Figure 4: Second split, $n=3, m=2: B_{2,0}=\operatorname{diag}(0,0, k-1), B_{2,1}=\operatorname{diag}(k, 0, k-1)$, $B_{2,2}=\operatorname{diag}(0, k, k-1), B_{2,3}=\operatorname{diag}(k, k, k-1)$.

supremum norm and Hölder norm, respectively, as

$$
\begin{aligned}
|f|_{C^{\alpha}(E)} & :=\sup _{\substack{x_{1}, x_{2} \in E \\
x_{1} \neq x_{2}}} \frac{\left|f\left(x_{2}\right)-f\left(x_{1}\right)\right|}{\left|x_{2}-x_{1}\right|^{\alpha}}, \quad\|f\|_{L^{\infty}(E)}=\sup _{x \in E}|f(x)|, \\
\|f\|_{C^{\alpha}(E)} & :=|f|_{C^{\alpha}(E)}+\|f\|_{L^{\infty}(E)} .
\end{aligned}
$$

We will write $f \in C^{\alpha}\left(E, \mathbb{R}^{n}\right)$ when $\|f\|_{C^{\alpha}(E)}<\infty$. Note that, if $f$ is continuous, the above norms and seminorms in $E$ coincide with those in $\bar{E}$. In particular, we will identify $C^{\alpha}\left(E, \mathbb{R}^{n}\right)$ with $C^{\alpha}\left(\bar{E}, \mathbb{R}^{n}\right)$, the set of Hölder functions of exponent $\alpha$. Of course, if $\alpha=1$, they are Lipschitz. A homeomorphism $f$ is bi-Hölder if both $f$ and $f^{-1}$ are Hölder.

The identity function is denoted by id.

We will say that a continuous mapping $f: \bar{\Omega} \rightarrow \mathbb{R}^{n}$ is piecewise affine if there exists a countable family $\left\{\Omega_{i}\right\}_{i \in \mathbb{N}}$ of pairwise disjoint open subsets of $\Omega$ such that $\left.f\right|_{\Omega_{i}}$ is affine for all $i \in \mathbb{N}$, and

$$
\left|\Omega \backslash \bigcup_{i \in \mathbb{N}} \Omega_{i}\right|=0 .
$$

\section{Approximation of laminates by functions}

The following result, based on Astala, Faraco and Székelyhidi [2, Prop. 2.3], shows how the gradient of a function can approximate a laminate. Given $A_{1}, A_{2} \in \Gamma_{+}$we write $A_{1} \geq A_{2}$ when $A_{1}-A_{2} \in \Gamma_{+}$. Note that, when $A \in \mathbb{R}^{n \times n}$, the same symbol $A$ is used to indicate a matrix (as in (E) below) and a linear function (as in (다)). 


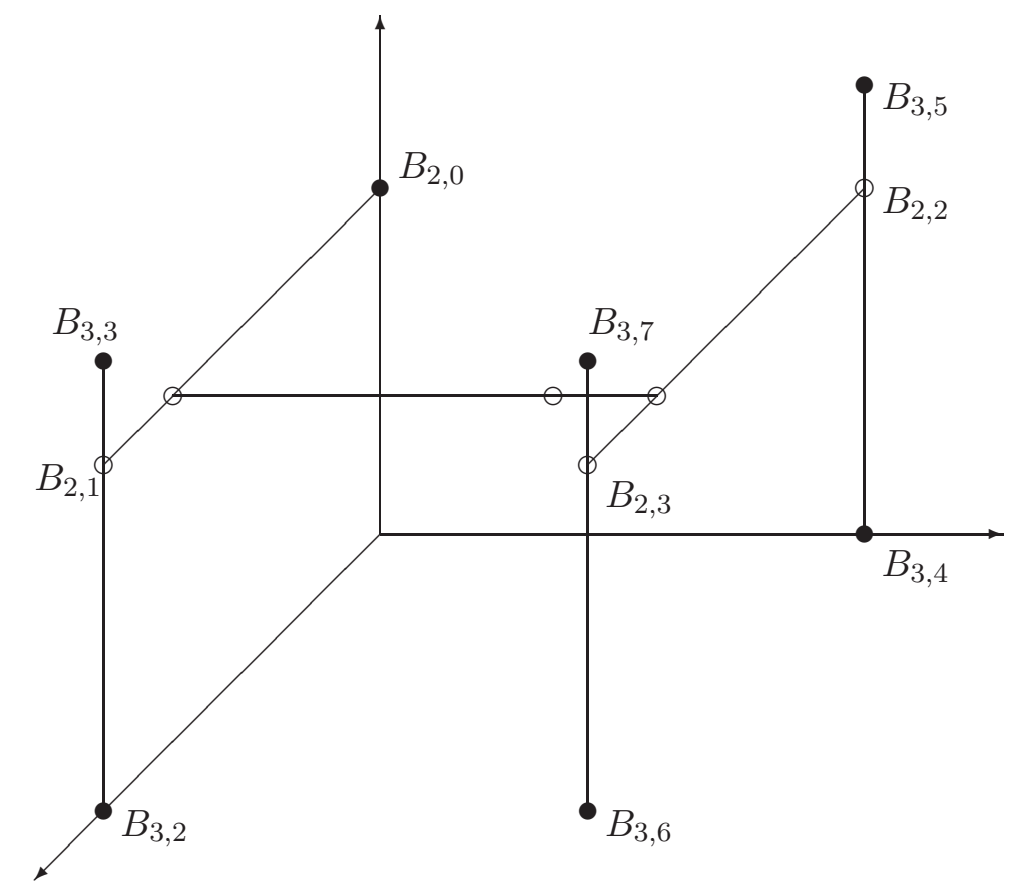

Figure 5: Third split, $n=3, m=2: B_{3,0}=B_{3,1}=B_{2,0}=\operatorname{diag}(0,0, k-1)$, $B_{3,2}=\operatorname{diag}(k, 0,0), B_{3,3}=\operatorname{diag}(k, 0, k), B_{3,4}=\operatorname{diag}(0, k, 0), B_{3,5}=\operatorname{diag}(0, k, k), B_{3,6}=$ $\operatorname{diag}(k, k, 0), B_{3,7}=k I$.

Proposition 3. Let $N \in \mathbb{N}, A_{1}, \ldots, A_{N} \in \Gamma_{+}$and $L \geq 1$ be such that

$$
A_{i} \geq L^{-1} I, \quad\left|A_{i}\right| \leq L, \quad i=1, \ldots, N .
$$

Consider $\alpha_{1}, \ldots, \alpha_{N} \geq 0$ such that $\nu:=\sum_{i=1}^{N} \alpha_{i} \delta_{A_{i}}$ is in $\mathcal{L}\left(\mathbb{R}^{n \times n}\right)$ and call $A:=\bar{\nu}$. Then, for every $\alpha \in(0,1), 0<\delta<\frac{1}{2} \min \left\{L^{-1}, \min _{1 \leq i<j \leq N}\left|A_{i}-A_{j}\right|\right\}$ and every bounded open set $\Omega \subset \mathbb{R}^{n}$, there exists a piecewise affine bi-Lipschitz homeomorphism $f: \Omega \rightarrow A \Omega$ such that

(a) $f=\nabla u$ for some $u \in W^{2, \infty}(\Omega)$,

(b) $f(x)=$ Ax for $x \in \partial \Omega$,

(c) $\|f-A\|_{C^{\alpha}(\bar{\Omega})}<\delta$,

(d) $\left\|f^{-1}-A^{-1}\right\|_{C^{\alpha}(A \bar{\Omega})}<\delta$,

(e) $\left|\left\{x \in \Omega:\left|D f(x)-A_{i}\right|<\delta\right\}\right|=\alpha_{i}|\Omega|$ for all $i=1, \ldots, N$.

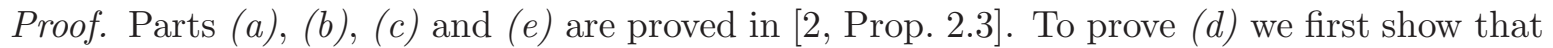
$f$ is bi-Lipschitz. We extend $f$ to an open ball $\Omega^{\prime}$ such that $\bar{\Omega} \subset \Omega^{\prime}$ and $f(x)=A x$ in $\Omega^{\prime} \backslash \Omega$. Thus, $f$ is continuous in $\Omega^{\prime}$. We define, for each $\varepsilon>0$,

$$
\Omega_{\varepsilon}^{\prime}=\left\{x \in \Omega^{\prime}: \operatorname{dist}\left(x, \partial \Omega^{\prime}\right)>\varepsilon\right\} .
$$

By (何) we get that $D f(x) \geq \frac{1}{2 L} I$, and $|D f(x)| \leq 2 L$ a.e. in $\Omega^{\prime}$. As $\Omega^{\prime}$ is convex then $f$ is $2 L$-Lipschitz. Let $\left\{\eta_{\varepsilon}\right\}_{0<\varepsilon \leq 1}$ be a standard family of mollifiers, and $f_{\varepsilon}:=\eta_{\varepsilon} * f \in C^{\infty}\left(\Omega_{\varepsilon}^{\prime}\right)$ the 
mollification of $f$. Using that the matrices $M \in \mathbb{R}_{\mathrm{sym}}^{n \times n}$ satisfying $(2 L)^{-1} I \leq M$ form a convex set, we find that there exists an $\varepsilon_{0}>0$ such that if $\varepsilon \leq \varepsilon_{0}$ then $D f_{\varepsilon}(x) \geq \frac{1}{2 L} I$ in $\Omega_{\varepsilon}^{\prime} \supset \bar{\Omega}$.

For each $x, y \in \Omega_{\varepsilon}^{\prime}$, calling $h=y-x$, we have that

$$
\begin{aligned}
\left|f_{\varepsilon}(y)-f_{\varepsilon}(x)\right||h| & \geq\left|\left\langle f_{\varepsilon}(y)-f_{\varepsilon}(x), h\right\rangle\right|=\left|\left\langle\int_{0}^{1} D f_{\varepsilon}(x+t h) h d t, h\right\rangle\right| \\
& \geq \int_{0}^{1}\left\langle D f_{\varepsilon}(x+t h) h, h\right\rangle d t \geq \int_{0}^{1} \frac{1}{2 L}|h|^{2} d t=\frac{1}{2 L}|h|^{2}
\end{aligned}
$$

that is,

$$
\left|f_{\varepsilon}(y)-f_{\varepsilon}(x)\right| \geq \frac{1}{2 L}|x-y|
$$

Using that $f_{\varepsilon} \rightarrow f$ uniformly in $\bar{\Omega}$ as $\varepsilon \rightarrow 0$, we get

$$
\frac{1}{2 L}|x-y| \leq|f(y)-f(x)|
$$

Hence $f$ is $K$-bi-Lipschitz in $\bar{\Omega}$ with $K=2 L$. Therefore, $f$ is a homeomorphism onto its image. The equalities $f(\Omega)=A \Omega$ and $f(\bar{\Omega})=A \bar{\Omega}$ follows from standard results using the topological degree (e.g., [3, Thms. 1 and 2$]$ ).

Now we will estimate the $C^{\alpha}$ seminorm of $f^{-1}-A^{-1}$. For each $y_{1}, y_{2} \in f(\bar{\Omega})$, let $x_{i}=$ $f^{-1}\left(y_{i}\right), i=1,2$. Using that $f$ is $K$-bilipschitz and that $A \geq L^{-1} I$, we get

$$
\begin{aligned}
\frac{\left|f^{-1}\left(y_{1}\right)-A^{-1} y_{1}-f^{-1}\left(y_{2}\right)+A^{-1} y_{2}\right|}{\left|y_{1}-y_{2}\right|^{\alpha}} & \leq K^{\alpha}\left|A^{-1}\right| \frac{\left|A x_{1}-f\left(x_{1}\right)-A x_{2}+f\left(x_{2}\right)\right|}{\left|x_{1}-x_{2}\right|^{\alpha}} \\
& \leq K^{\alpha}\left|A^{-1}\right||f-A|_{C^{\alpha}(\bar{\Omega})} \leq K^{\alpha} L \delta=2 L^{\alpha+1} \delta
\end{aligned}
$$

so

$$
\left|f^{-1}-A^{-1}\right|_{C^{\alpha}(A \bar{\Omega})} \leq 2 \delta L^{\alpha+1} .
$$

Therefore, $\left|f^{-1}-A^{-1}\right|_{C^{\alpha}(A \bar{\Omega})}$ can be done as small as we wish. Finally,

$$
\left\|f^{-1}-A^{-1}\right\|_{L^{\infty}(A \bar{\Omega})} \leq\left|f^{-1}-A^{-1}\right|_{C^{\alpha}(A \bar{\Omega})}(\operatorname{diam} A \Omega)^{\alpha}
$$

so $\left\|f^{-1}-A^{-1}\right\|_{C^{\alpha}(A \bar{\Omega})}$ is as small as we wish.

\section{Cutting and pasting Hölder homeomorphisms}

In this section we prove that if we modify a bi-Hölder homeomorphism in some sets by cutting and pasting other bi-Hölder homeomorphisms, the modified map is still a bi-Hölder homeomorphism.

First we show how to bound the $C^{\alpha}$ norm of a function in $\Omega$ with its $C^{\alpha}$ norms in a collection of subsets of $\Omega$ that covers $\Omega$ up to measure zero. 
Lemma 4. Let $\alpha \in(0,1],\left\{\Omega_{i}\right\}_{i=1}^{\infty} \subset \Omega$ pairwise disjoint open sets such that $\left|\Omega \backslash \bigcup_{i=1}^{\infty} \Omega_{i}\right|=0$, and $f: \bar{\Omega} \rightarrow \mathbb{R}^{n}$ such that $f(x)=0$ for all $x \in \bigcup_{i=1}^{\infty} \partial \Omega_{i}$. Then

$$
\|f\|_{C^{\alpha}(\bar{\Omega})} \leq 2 \sup _{i \in \mathbb{N}}\|f\|_{C^{\alpha}\left(\overline{\Omega_{i}}\right)}
$$

Proof. We assume that the right hand side of the last inequality is finite. Given $x, y \in \bigcup_{i=1}^{\infty} \overline{\Omega_{i}}$, let $i_{0}, i_{1} \in \mathbb{N}$ be such that $x \in \overline{\Omega_{i_{0}}}$ and $y \in \overline{\Omega_{i_{1}}}$.

If $i_{0}=i_{1}$, then $|f(x)-f(y)| \leq|f|_{C^{\alpha}\left(\overline{\Omega_{i_{0}}}\right)}|x-y|^{\alpha}$. Whereas if $i_{0} \neq i_{1}$, we have $\Omega_{i_{0}} \cap \Omega_{i_{1}}=\emptyset$. Consider

$$
\lambda_{0}:=\min \left\{\lambda \in[0,1]: x+\lambda(y-x) \in \partial \Omega_{i_{0}}\right\}, \quad \lambda_{1}:=\max \left\{\lambda \in[0,1]: x+\lambda(y-x) \in \partial \Omega_{i_{1}}\right\}
$$

and denote $x^{\prime}=x+\lambda_{0}(y-x)$ and $y^{\prime}=x+\lambda_{1}(y-x)$. Then, $f\left(x^{\prime}\right)=f\left(y^{\prime}\right)=0$, and, therefore,

$$
\begin{aligned}
|f(x)-f(y)| & \leq\left|f(x)-f\left(x^{\prime}\right)\right|+\left|f\left(y^{\prime}\right)-f(y)\right| \leq|f|_{C^{\alpha}\left(\overline{\Omega_{i_{0}}}\right)}\left|x-x^{\prime}\right|^{\alpha}+|f|_{C^{\alpha}\left(\overline{\Omega_{i_{1}}}\right)}\left|y^{\prime}-y\right|^{\alpha} \\
& \leq\left(|f|_{C^{\alpha}\left(\overline{\Omega_{i_{0}}}\right)}+|f|_{C^{\alpha}\left(\overline{\Omega_{i_{1}}}\right)}\right)|x-y|^{\alpha} .
\end{aligned}
$$

On the other hand, $\|f\|_{L^{\infty}\left(\bigcup_{i=1}^{\infty} \overline{\Omega_{i}}\right)}=\sup _{i \in \mathbb{N}}\|f\|_{L^{\infty}\left(\overline{\Omega_{i}}\right)}$. Hence,

$$
\|f\|_{C^{\alpha}\left(\bigcup_{i=1}^{\infty} \overline{\Omega_{i}}\right)} \leq 2 \sup _{i \in \mathbb{N}}\|f\|_{C^{\alpha}\left(\overline{\Omega_{i}}\right)}
$$

As $\bigcup_{i=1}^{\infty} \overline{\Omega_{i}}$ is dense in $\bar{\Omega}$, the required bound holds due to the uniform continuity.

The main result of this section is the following.

Lemma 5. Let $f: \bar{\Omega} \rightarrow \mathbb{R}^{n}$ be a homeomorphism such that $f$ and $f^{-1}$ are $C^{\alpha}$ for some $\alpha \in(0,1]$. Let $\left\{\omega_{i}\right\}_{i \in \mathbb{N}} \subset \Omega$ be a family of pairwise disjoint open sets, and for each $i \in \mathbb{N}$ let $g_{i}: \overline{\omega_{i}} \rightarrow f\left(\overline{\omega_{i}}\right)$ be a homeomorphism such that $g_{i}=f$ on $\partial \omega_{i}$,

$$
\sup _{i \in \mathbb{N}}\left\|f-g_{i}\right\|_{C^{\alpha}\left(\overline{\omega_{i}}\right)}<\infty \quad \text { and } \quad \sup _{i \in \mathbb{N}}\left\|f^{-1}-g_{i}^{-1}\right\|_{C^{\alpha}\left(f\left(\overline{\omega_{i}}\right)\right)}<\infty .
$$

Then, the function

$$
\tilde{f}(x):= \begin{cases}f(x) & \text { if } x \in \bar{\Omega} \backslash \bigcup_{i \in \mathbb{N}} \omega_{i}, \\ g_{i}(x) & \text { if } x \in \omega_{i} \text { for some } i \in \mathbb{N}\end{cases}
$$

is a homeomorphism between $\bar{\Omega}$ and $f(\bar{\Omega})$ such that $\tilde{f}$ and $\tilde{f}^{-1}$ are $C^{\alpha}$ and

$$
\|f-\tilde{f}\|_{C^{\alpha}(\bar{\Omega})} \leq 2 \sup _{i \in \mathbb{N}}\left\|f-g_{i}\right\|_{C^{\alpha}\left(\overline{\omega_{i}}\right)}, \quad\left\|f^{-1}-\tilde{f}^{-1}\right\|_{C^{\alpha}(\bar{\Omega})} \leq 2 \sup _{i \in \mathbb{N}}\left\|f^{-1}-g_{i}^{-1}\right\|_{C^{\alpha}\left(f\left(\overline{\omega_{i}}\right)\right)} .
$$

Proof. Using that $f$ and $g_{i}$ are homeomorphisms we have that $f\left(\omega_{i}\right)=g_{i}\left(\omega_{i}\right)$, for each $i \in \mathbb{N}$. Thus, it is clear that the function

$$
f(\bar{\Omega}) \ni y \longmapsto \begin{cases}f^{-1}(y) & \text { if } y \in f\left(\bar{\Omega} \backslash \bigcup_{i \in \mathbb{N}} \omega_{i}\right), \\ g_{i}^{-1}(y) & \text { if } y \in f\left(\omega_{i}\right) \text { for some } i \in \mathbb{N}\end{cases}
$$


is the inverse of $\tilde{f}$.

Using Lemma 4, we obtain that

$$
\begin{aligned}
& \|\tilde{f}-f\|_{C^{\alpha}\left(\overline{\bigcup_{i \in \mathbb{N}} \omega_{i}}\right)} \leq 2 \sup _{i \in \mathbb{N}}\left\|f-g_{i}\right\|_{C^{\alpha}\left(\overline{\omega_{i}}\right)}, \\
& \left\|\tilde{f}^{-1}-f^{-1}\right\|_{C^{\alpha}\left(f\left(\overline{\bigcup_{i \in \mathbb{N}} \omega_{i}}\right)\right)} \leq 2 \sup _{i \in \mathbb{N}}\left\|f^{-1}-g_{i}^{-1}\right\|_{C^{\alpha}\left(f\left(\overline{\omega_{i}}\right)\right) .}
\end{aligned}
$$

Moreover, when we call $F:=\tilde{f}-f$, we have that $F=0$ in $\bar{\Omega} \backslash \bigcup_{i \in \mathbb{N}} \omega_{i}$. In order to show that $F$ is $C^{\alpha}$ in $\bar{\Omega}$, given $x_{1} \in \bar{\Omega} \backslash \bigcup_{i \in \mathbb{N}} \omega_{i}$ and $x_{2} \in \bigcup_{i \in \mathbb{N}} \omega_{i}$, we take $x_{3}=x_{1}+\lambda\left(x_{2}-x_{1}\right)$ for some $\lambda \in[0,1]$ such that $x_{3} \in \partial \bigcup_{i \in \mathbb{N}} \omega_{i}$. Then $F\left(x_{1}\right)=F\left(x_{3}\right)=0$ and, hence,

$$
\left|F\left(x_{2}\right)-F\left(x_{1}\right)\right|=\left|F\left(x_{3}\right)-F\left(x_{1}\right)\right| \leq\|F\|_{C^{\alpha}\left(\overline{\bigcup_{i \in \mathbb{N}} \omega_{i}}\right)}\left|x_{3}-x_{1}\right|^{\alpha} \leq\|F\|_{C^{\alpha}\left(\overline{\bigcup_{i \in \mathbb{N}} \omega_{i}}\right)}\left|x_{2}-x_{1}\right|^{\alpha} .
$$

This shows that $F \in C^{\alpha}\left(\bar{\Omega}, \mathbb{R}^{n}\right)$. Analogously, $\tilde{f}^{-1}-f^{-1}$ is $C^{\alpha}$ in $f(\bar{\Omega})$ and the last bound of the statement also holds. In particular, $\tilde{f}$ and $\tilde{f}^{-1}$ are $C^{\alpha}$, and, hence, $\tilde{f}$ is a homeomorphism between $\bar{\Omega}$ and $f(\bar{\Omega})$.

\section{Construction of the laminate and its approximation}

This section constructs the sequence of laminates together with their approximations by functions. We will continuously use the following sets and constants.

For $j \in \mathbb{N}$, we define the sequence of open sets $E_{j}$ by

$E_{j}=\left\{A \in \Gamma_{+}:|A|>\frac{1}{2}+2^{-j}, 2^{-j-m}<\sigma_{i}(A) \max \left\{|A|^{m-1}, 1\right\}<2^{-j}\right.$ for $\left.1 \leq i \leq n-m+1\right\}$.

For $a \in \mathbb{N}, 0 \leq a \leq n-m, j \in \mathbb{N}$ and $\mathcal{R}>\frac{1}{2}+2^{-j}$ such that

$$
\rho_{j, \mathcal{R}}:=\frac{3 \cdot 2^{-j-2}}{\max \left\{\mathcal{R}^{m-1}, 1\right\}}<\mathcal{R},
$$

we define the closed sets

$E_{j, \mathcal{R}}^{a}=\left\{A \in \Gamma_{+}:|A|>\frac{1}{2}+2^{-j}, \quad \sigma_{i}(A)=\rho_{j, \mathcal{R}}\right.$ for $1 \leq i \leq a, \quad \sigma_{i}(A)=\mathcal{R}$ for $\left.a+1 \leq i \leq n\right\}$.

We also denote

$$
E_{j}^{a}=\bigcup_{\mathcal{R} \in\left(\frac{1}{2}+2^{-j}, \infty\right)} E_{j, \mathcal{R}}^{a} .
$$

The sets $E_{j}$ approximate the set of positive semidefinite matrices with rank less than $m$, and the sets $E_{j, \mathcal{R}}^{a}$ approximate the set

$$
\left\{\mathcal{R} Q I_{a} Q^{T}: Q \in S O(n)\right\}, \quad I_{a}=\operatorname{diag}(\underbrace{0, \ldots, 0}_{a}, \underbrace{1 \ldots, 1}_{n-a}) .
$$


The number $\mathcal{R}$ plays the role of $k$ in Section 2 and eventually will tend to infinity. The number $\rho_{j, \mathcal{R}}$ will tend to zero: the reason that it appears in the definition of $E_{j, \mathcal{R}}^{a}$ is that, even though $E_{j, \mathcal{R}}^{a}$ approximates a subset of matrices of rank $n-a$, we need them to be invertible.

Given $j \in \mathbb{N}$ and $\mathcal{R}>\frac{1}{2}+2^{-j}$ we define

$$
\begin{aligned}
r_{j, \mathcal{R}}:=\frac{1}{2} \min \left\{1-\left(\frac{2}{3}\right)^{\frac{1}{m-1}}, \rho_{j, \mathcal{R}}\left(1-\frac{\max \left\{1, \mathcal{R}^{m-1}\right\}}{(\mathcal{R}+1)^{m-1}}\right), \frac{\rho_{j, \mathcal{R}}}{3}, \mathcal{R}-\frac{1}{2}-2^{-j},\right. \\
\left.\frac{1}{2^{n}}(\mathcal{R}+1)^{-m-1}, \frac{1-\rho_{j, \mathcal{R}+1}}{\max \{1, \mathcal{R}\}}\right\}
\end{aligned}
$$

and for $j \in \mathbb{N}, a_{0}, a \in\{0, \ldots, n-m\}, \mathcal{R}>\rho_{j, \mathcal{R}}$ we denote

$$
\begin{aligned}
C\left(j, \mathcal{R}, a_{0}, a\right)= & \sum_{b=} \sum^{\min \left\{a_{0}, a\right\}}\left(\begin{array}{c}
a_{0} \\
b
\end{array}\right)\left(\begin{array}{c}
\left.n-a_{0}+a-n\right\} \\
a-b
\end{array}\right)\left(\frac{\mathcal{R}+r_{j, \mathcal{R}}}{\mathcal{R}+1}\right)^{n-a_{0}-a+b} \\
& \left(\frac{1}{\max \{1, \mathcal{R}\}}\right)^{a-b}\left(\frac{2^{-j}}{(\mathcal{R}+1) \max \left\{1, \mathcal{R}^{m-1}\right\}}\right)^{a_{0}-b} .
\end{aligned}
$$

We have chosen $r_{j, \mathcal{R}}$ small enough, depending on $\mathcal{R}$ (and, hence, on $\rho_{j, \mathcal{R}}$ ) to perform all the computations in this section: it will play the role of the $\delta$ of Proposition 3 .

The next lemma constructs a laminate with the required integrability. The second part of its proof follows that of Section 2

Lemma 6. Let $j \in \mathbb{N}, a_{0} \in\{0, \ldots, n-m\}, \mathcal{R}>0$ with $\rho_{j, \mathcal{R}}<\mathcal{R}$ and $A \in \Gamma_{+}$be such that $\operatorname{dist}\left(A, E_{j, \mathcal{R}}^{a_{0}}\right)<r_{j, \mathcal{R}}$. Then there exists $\nu \in \mathcal{L}\left(\mathbb{R}^{n \times n}\right)$ such that $\bar{\nu}=A$,

$$
\operatorname{supp} \nu \subset\left(\bigcup_{a=0}^{n-m} E_{j, \mathcal{R}+1}^{a} \cup E_{j}\right) \cap\left\{\xi \in \mathbb{R}^{n \times n}:|\xi| \leq \mathcal{R}+1\right\}
$$

and for $0 \leq a \leq n-m$,

$$
\nu\left(E_{j, \mathcal{R}+1}^{a}\right) \leq C\left(j, \mathcal{R}, a_{0}, a\right) .
$$

Proof. There exist $Q \in S O(n)$ and $B \in E_{j, \mathcal{R}}^{a_{0}}$ such that $A=Q \operatorname{diag}\left(\sigma_{1}, \ldots, \sigma_{n}\right) Q^{T}$, with $0<\sigma_{1} \leq \cdots \leq \sigma_{n}$ and $|A-B|<r_{j, \mathcal{R}}$. Using the inequality

$$
\left|\sigma_{i}(A)-\sigma_{i}(B)\right| \leq|A-B|, \quad i=1, \ldots, n,
$$

(see, e.g., [18, Cor. 4.5]), we find that

$$
\left|\sigma_{i}-\rho_{j, \mathcal{R}}\right|<r_{j, \mathcal{R}} \quad \text { for } 1 \leq i \leq a_{0}, \quad \text { and } \quad\left|\sigma_{i}-\mathcal{R}\right|<r_{j, \mathcal{R}} \quad \text { for } a_{0}+1 \leq i \leq n .
$$

In order to construct the desired laminate we prove that:

1) $\sigma_{n}<\mathcal{R}+1$. 
2) $\rho_{j, \mathcal{R}+1}<\sigma_{1}$.

3) $2^{-j-m}<\rho_{j, \mathcal{R}+1} \max \left\{1, \sigma_{n}^{m-1}\right\}<2^{-j}$.

Inequality (1) is obvious thanks to (24) since $r_{j, \mathcal{R}}<1$. By (24), the definition of $\rho_{j, \mathcal{R}+1}$ and (21) we obtain

$$
\rho_{j, \mathcal{R}+1}=\frac{3 \cdot 2^{-j-2}}{(\mathcal{R}+1)^{m-1}}=\frac{\rho_{j, \mathcal{R}} \max \left\{1, \mathcal{R}^{m-1}\right\}}{(\mathcal{R}+1)^{m-1}}<\rho_{j, \mathcal{R}}-r_{j, \mathcal{R}}<\sigma_{1},
$$

where in the last inequality we have differentiated the cases $a_{0}=0$ and $a_{0}>0$. So we have 2). Lastly, we prove 31). On the one hand,

$\rho_{j, \mathcal{R}+1} \max \left\{1, \sigma_{n}^{m-1}\right\} \leq \rho_{j, \mathcal{R}+1} \max \left\{1,\left(\mathcal{R}+r_{j, \mathcal{R}}\right)^{m-1}\right\} \leq \rho_{j, \mathcal{R}+1}(\mathcal{R}+1)^{m-1}=3 \cdot 2^{-j-2}<2^{-j}$

and, on the other hand,

$\rho_{j, \mathcal{R}+1} \max \left\{1, \sigma_{n}^{m-1}\right\} \geq \rho_{j, \mathcal{R}+1} \max \left\{1,\left(\mathcal{R}-r_{j, \mathcal{R}}\right)^{m-1}\right\}=\frac{3 \cdot 2^{-j-2} \max \left\{1,\left(\mathcal{R}-r_{j, \mathcal{R}}\right)^{m-1}\right\}}{(\mathcal{R}+1)^{m-1}}$.

Therefore, if $\mathcal{R} \leq 1$ we have

$$
\frac{3 \cdot 2^{-j-2} \max \left\{1,\left(\mathcal{R}-r_{j, \mathcal{R}}\right)^{m-1}\right\}}{(\mathcal{R}+1)^{m-1}} \geq 3 \cdot 2^{-j-m-1}>2^{-j-m},
$$

whereas if $\mathcal{R}>1$ we use (21) to obtain

$3 \cdot 2^{-j-2} \frac{\max \left\{1,\left(\mathcal{R}-r_{j, \mathcal{R}}\right)^{m-1}\right\}}{(\mathcal{R}+1)^{m-1}} \geq 3 \cdot 2^{-j-2} \frac{\left(\mathcal{R}-r_{j, \mathcal{R}}\right)^{m-1}}{(\mathcal{R}+1)^{m-1}} \geq 3 \cdot 2^{-j-m-1}\left(1-r_{j, \mathcal{R}}\right)^{m-1}>2^{-j-m}$.

Thus, 3) is proved.

Now we build the laminate, following the lines of Section 2. We shall construct families

$$
\left\{B_{\ell, i}\right\}_{\substack{\ell=0, \ldots, n \\ i=0, \ldots 2^{\ell}-1}} \subset \Gamma_{+} \quad \text { and } \quad\left\{\lambda_{\ell, i}\right\}_{\substack{\ell=0, \ldots, n \\ i=0, \ldots 2^{\ell}-1}} \subset[0,1]
$$

by finite induction on $\ell$. Let $B_{0,0}=A, \lambda_{0,0}=1$ and for $0 \leq \ell \leq n-1,0 \leq i \leq 2^{\ell}-1$, we assume $\left\{B_{\ell, i}\right\}_{i=0}^{2^{\ell}-1}$ and $\left\{\lambda_{\ell, i}\right\}_{i=0}^{2^{\ell}-1}$ have been defined, $Q^{T} B_{\ell, j} Q$ is diagonal, $\lambda_{\ell, i} \geq 0$,

$$
\sum_{i=0}^{2^{\ell}-1} \lambda_{\ell, i}=1, \quad B_{0,0}=\sum_{i=0}^{2^{\ell}-1} \lambda_{\ell, i} B_{\ell, i}, \quad \sum_{i=0}^{2^{\ell}-1} \lambda_{\ell, i} \delta_{B_{\ell, i}} \in \mathcal{L}\left(\mathbb{R}^{n \times n}\right)
$$

and

$$
\left(Q^{T} B_{\ell, i} Q\right)_{\alpha, \alpha}=\sigma_{\alpha} \quad \text { if } \alpha=\ell+1, \ldots, n \text {. }
$$


We also assume that if $B_{\ell, i} \notin E_{j}$ then

$$
\left(Q^{T} B_{\ell, i} Q\right)_{\alpha, \alpha} \in\left\{\rho_{j, \mathcal{R}+1}, \mathcal{R}+1\right\}, \quad \alpha=1, \ldots, \ell,
$$

and when we let

$$
\begin{gathered}
\beta_{\ell, i}:=\#\left\{\alpha \in\left\{1, \ldots, \min \left\{a_{0}, \ell\right\}\right\}:\left(Q^{T} B_{\ell, i} Q\right)_{\alpha, \alpha}=\rho_{j, \mathcal{R}+1}\right\}, \\
\gamma_{\ell, i}:=\#\left\{\alpha \in\left\{a_{0}+1, \ldots, \ell\right\}:\left(Q^{T} B_{\ell, i} Q\right)_{\alpha, \alpha}=\rho_{j, \mathcal{R}+1}\right\},
\end{gathered}
$$

then

$$
\beta_{\ell, i}+\gamma_{\ell, i} \leq n-m
$$

and, calling

$$
U:=\frac{2^{-j}}{(\mathcal{R}+1) \max \left\{1, \mathcal{R}^{m-1}\right\}}, \quad V:=\frac{1}{\max \{1, \mathcal{R}\}}, \quad W:=\frac{\mathcal{R}+r_{j, \mathcal{R}}}{\mathcal{R}+1},
$$

we have

$$
\lambda_{\ell, i} \leq U^{\min \left\{a_{0}, \ell\right\}-\beta_{\ell, i}} V^{\gamma_{\ell, i}} W^{\max \left\{0, \ell-a_{0}-\gamma_{\ell, i}\right\}} .
$$

We assume additionally that for each $B_{\ell, i^{\prime}} \notin E_{j}$ such that $i^{\prime} \neq i$, we have $B_{\ell, i^{\prime}} \neq B_{\ell, i}$.

With the above induction hypotheses, we construct $\left\{B_{\ell+1, i}\right\}_{i=0}^{2^{\ell+1}-1}$ and $\left\{\lambda_{\ell+1, i}\right\}_{i=0}^{2^{\ell+1}-1}$ as follows. For any $0 \leq i \leq 2^{\ell}-1$, define

$$
\begin{gathered}
B_{\ell+1,2 i}=\left\{\begin{array}{lr}
B_{\ell, i}-Q \operatorname{diag}(\underbrace{0, \ldots, 0}_{\ell}, \sigma_{\ell+1}-\rho_{j, \mathcal{R}+1}, \underbrace{0, \ldots, 0}_{n-\ell-1}) Q^{T}, & \text { if } B_{\ell, i} \notin E_{j}, \\
B_{\ell, i}, & \text { if } B_{\ell, i} \in E_{j},
\end{array}\right. \\
B_{\ell+1,2 i+1}= \begin{cases}B_{\ell, i}+Q \operatorname{diag}(\underbrace{0, \ldots, 0}_{\ell}, \mathcal{R}+1-\sigma_{\ell+1}, \underbrace{0, \ldots, 0}_{n-\ell-1}) Q^{T}, & \text { if } B_{\ell, i} \notin E_{j}, \\
B_{\ell, i}, & \text { if } B_{\ell, i} \in E_{j}, \\
\lambda_{\ell+1,2 i}=\lambda_{\ell, i} \frac{\mathcal{R}+1-\sigma_{\ell+1}}{\mathcal{R}+1-\rho_{j, \mathcal{R}+1}} \text { and } \quad \lambda_{\ell+1,2 i+1}=\lambda_{\ell, i} \frac{\sigma_{\ell+1}-\rho_{j, \mathcal{R}+1}}{\mathcal{R}+1-\rho_{j, \mathcal{R}+1} .}\end{cases}
\end{gathered}
$$

So $\operatorname{rank}\left(B_{\ell+1,2 i}-B_{\ell+1,2 i+1}\right) \leq 1, \lambda_{\ell+1,2 i} \geq 0$ by 11), $\lambda_{\ell+1,2 i+1} \geq 0$ by 21), and

$$
B_{\ell, i}=\frac{\mathcal{R}+1-\sigma_{\ell+1}}{\mathcal{R}+1-\rho_{j, \mathcal{R}+1}} B_{\ell+1,2 i}+\frac{\sigma_{\ell+1}-\rho_{j, \mathcal{R}+1}}{\mathcal{R}+1-\rho_{j, \mathcal{R}+1}} B_{\ell+1,2 i+1} .
$$

With this, we can easily see that properties (26) hold for $\ell+1$. In what follows, $0 \leq i \leq 2^{\ell+1}-1$. We have

$$
\left(Q^{T} B_{\ell+1, i} Q\right)_{\alpha, \alpha}=\left(Q^{T} B_{\ell,\left\lfloor\frac{i}{2}\right\rfloor} Q\right)_{\alpha, \alpha}, \quad \alpha \neq \ell+1
$$




$$
\left(Q^{T} B_{\ell+1, i} Q\right)_{\ell+1, \ell+1}= \begin{cases}\rho_{j, \mathcal{R}+1}, & \text { if } B_{\ell,\left\lfloor\frac{i}{2}\right\rfloor} \notin E_{j}, i \text { even } \\ \mathcal{R}+1, & \text { if } B_{\ell,\left\lfloor\frac{i}{2}\right\rfloor} \notin E_{j}, i \text { odd } \\ \left(Q^{T} B_{\ell,\left\lfloor\frac{i}{2}\right\rfloor} Q\right)_{\ell+1, \ell+1}, & \text { if } B_{\ell,\left\lfloor\frac{i}{2}\right\rfloor} \in E_{j} .\end{cases}
$$

Therefore, property (27) holds for $\ell+1$. Now fix $\ell, i$ such that $B_{\ell+1, i} \notin E_{j}$. Then $B_{\ell+1,\left\lfloor\frac{i}{2}\right\rfloor} \notin E_{j}$, property (28) holds for $\ell+1$, and

$$
\beta_{\ell+1, i}=\left\{\begin{array}{ll}
\beta_{\ell, \frac{i}{2}}+1 & \text { if } i \text { is even, } \ell<a_{0}, \\
\beta_{\ell, \frac{i}{2}} & \text { if } i \text { is even, } \ell \geq a_{0}, \\
\beta_{\ell, \frac{i-1}{2}} & \text { if } i \text { is odd, }
\end{array} \quad \gamma_{\ell+1, i}= \begin{cases}\gamma_{\ell, \frac{i}{2}} & \text { if } i \text { is even, } \ell<a_{0}, \\
\gamma_{\ell, \frac{i}{2}}+1 & \text { if } i \text { is even, } \ell \geq a_{0}, \\
\gamma_{\ell, \frac{i-1}{2}} & \text { if } i \text { is odd. }\end{cases}\right.
$$

Using (29) we find that $\beta_{\ell+1, i}+\gamma_{\ell+1, i} \leq n-m+1$. On the other hand, we have shown that

$$
\sigma_{\alpha}\left(B_{\ell+1, i}\right) \in\left\{\rho_{j, \mathcal{R}+1}, \mathcal{R}+1, \sigma_{\ell+2}, \ldots, \sigma_{n}\right\}, \quad \alpha=1, \ldots, n .
$$

Thus, if we had $\beta_{\ell+1, i}+\gamma_{\ell+1, i}=n-m+1$ then, by 10) and 21) we would get

$$
\sigma_{\alpha}\left(B_{\ell+1, i}\right)=\rho_{j, \mathcal{R}+1}, \quad \alpha=1, \ldots, n-m+1 .
$$

and by [3), $B_{\ell+1, i} \in E_{j}$, which is a contradiction. Therefore, (29) holds for $\ell+1$.

Now let $i^{\prime} \neq i$ be such that $B_{\ell+1, i^{\prime}} \notin E_{j}$. If $\left\lfloor\frac{i}{2}\right\rfloor \neq\left\lfloor\frac{i^{\prime}}{2}\right\rfloor$, then $B_{\ell,\left\lfloor\frac{i^{\prime}}{2}\right\rfloor} \neq B_{\ell,\left\lfloor\frac{i}{2}\right\rfloor}$, and, hence, $B_{\ell+1, i^{\prime}} \neq B_{\ell+1, i}$, whereas if $\left\lfloor\frac{i}{2}\right\rfloor=\left\lfloor\frac{i^{\prime}}{2}\right\rfloor$, then $\left(B_{\ell+1, i^{\prime}}\right)_{\ell+1, \ell+1} \neq\left(B_{\ell+1, j}\right)_{\ell+1, \ell+1}$, and, hence, $B_{\ell+1, i^{\prime}} \neq B_{\ell+1, i}$.

Now we bound $\lambda_{\ell+1, i}$. Recall the notation (30) and the induction hypothesis (31). If $i$ is even and $\ell<a_{0}$, we have $\max \left\{0, \ell+1-a_{0}-\gamma_{\ell, i}\right\}=0$ and, therefore,

$$
\begin{aligned}
\lambda_{\ell+1, i} & =\lambda_{\ell, \frac{i}{2}} \frac{\mathcal{R}+1-\sigma_{\ell+1}}{\mathcal{R}+1-\rho_{j, \mathcal{R}+1}} \leq \lambda_{\ell, \frac{i}{2}} \leq U^{\min \left\{a_{0}, \ell\right\}-\beta_{\ell, \frac{i}{2}}} V^{\gamma_{\ell, \frac{i}{2}}} W^{\max \left\{0, \ell-a_{0}-\gamma_{\ell, \frac{i}{2}}\right\}} \\
& =U^{\min \left\{a_{0}, \ell+1\right\}-\beta_{\ell+1, i}} V^{\gamma_{\ell+1, i}} W^{\max \left\{0, \ell+1-a_{0}-\gamma_{\ell+1, i}\right\}} .
\end{aligned}
$$

If $i$ is even and $\ell \geq a_{0}$, using (24) and (21), we have

$$
\frac{\mathcal{R}+1-\sigma_{\ell+1}}{\mathcal{R}+1-\rho_{j, \mathcal{R}+1}} \leq \frac{1+r_{j, \mathcal{R}}}{\mathcal{R}+1-\rho_{j, \mathcal{R}+1}} \leq \frac{1}{\max \{1, \mathcal{R}\}}
$$

therefore

$$
\begin{aligned}
\lambda_{\ell+1, i} & =\lambda_{\ell, \frac{i}{2}} \frac{\mathcal{R}+1-\sigma_{\ell+1}}{\mathcal{R}+1-\rho_{j, \mathcal{R}+1}} \leq \lambda_{\ell, \frac{i}{2}} \frac{1}{\max \{1, \mathcal{R}\}} \leq U^{\min \left\{a_{0}, \ell\right\}-\beta_{\ell, \frac{i}{2}}} V^{\gamma_{\ell, \frac{i}{2}}+1} W^{\max \left\{0, \ell-a_{0}-\gamma_{\ell, \frac{i}{2}}\right\}} \\
& =U^{\min \left\{a_{0}, \ell+1\right\}-\beta_{\ell+1, i}} V^{\gamma_{\ell+1, i}} W^{\max \left\{0, \ell+1-a_{0}-\gamma_{\ell+1, i}\right\}} .
\end{aligned}
$$

If $i$ is odd and $\ell<a_{0}$, then, by (24) and the definition of $r_{j, \mathcal{R}}$ and $\rho_{j, \mathcal{R}}$, we have

$$
\frac{\sigma_{\ell+1}-\rho_{j, \mathcal{R}+1}}{\mathcal{R}+1-\rho_{j, \mathcal{R}+1}} \leq \frac{\rho_{j, \mathcal{R}}+r_{j, \mathcal{R}}-\rho_{j, \mathcal{R}+1}}{\mathcal{R}+1-\rho_{j, \mathcal{R}+1}} \leq \frac{\rho_{j, \mathcal{R}}+r_{j, \mathcal{R}}}{\mathcal{R}+1} \leq \frac{4 \rho_{j, \mathcal{R}}}{3(\mathcal{R}+1)} \leq \frac{2^{-j}}{(\mathcal{R}+1) \max \left\{1, \mathcal{R}^{m-1}\right\}}
$$


and

$$
\begin{aligned}
\lambda_{\ell+1, i} & =\lambda_{\ell, \frac{i-1}{2}} \frac{\sigma_{\ell+1}-\rho_{j, \mathcal{R}+1}}{\mathcal{R}+1-\rho_{j, \mathcal{R}+1}} \leq \lambda_{\ell, \frac{i-1}{2}} U \leq U^{\min \left\{a_{0}, \ell\right\}-\beta_{\ell, \frac{i-1}{2}+1}} V^{\gamma_{\ell, \frac{i-1}{2}}} W^{\max \left\{0, \ell-a_{0}-\gamma_{\ell, \frac{i-1}{2}}\right\}} \\
& =U^{\min \left\{a_{0}, \ell+1\right\}-\beta_{\ell+1, i}} V^{\gamma_{\ell+1, i}} W^{\max \left\{0, \ell+1-a_{0}-\gamma_{\ell+1, i}\right\}} .
\end{aligned}
$$

Finally, if $i$ is odd and $\ell \geq a_{0}$ we have $\gamma_{\ell, i} \leq \ell-a_{0}$ for all $i=0, \ldots, 2^{\ell}-1$, and

$$
\frac{\sigma_{\ell+1}-\rho_{j, \mathcal{R}+1}}{\mathcal{R}+1-\rho_{j, \mathcal{R}+1}} \leq \frac{\mathcal{R}+r_{j, \mathcal{R}}-\rho_{j, \mathcal{R}+1}}{\mathcal{R}+1-\rho_{j, \mathcal{R}+1}} \leq \frac{\mathcal{R}+r_{j, \mathcal{R}}}{\mathcal{R}+1}
$$

so

$$
\begin{aligned}
\lambda_{\ell+1, i} & =\lambda_{\ell, \frac{i-1}{2}} \frac{\sigma_{\ell+1}-\rho_{j, \mathcal{R}+1}}{\mathcal{R}+1-\rho_{j, \mathcal{R}+1}} \leq \lambda_{\ell, \frac{i-1}{2}} W \leq U^{\min \left\{a_{0}, \ell\right\}-\beta_{\ell, \frac{i-1}{2}}} V^{\gamma_{\ell, \frac{i-1}{2}}} W^{\max \left\{0, \ell-a_{0}-\gamma_{\ell, \frac{i-1}{2}}\right\}+1} \\
& =U^{\min \left\{a_{0}, \ell+1\right\}-\beta_{\ell+1, i}} V^{\gamma_{\ell+1, i}} W^{\max \left\{0, \ell+1-a_{0}-\gamma_{\ell+1, i}\right\}} .
\end{aligned}
$$

With this, we finish the inductive construction of the families (25). In particular, for all $0 \leq i \leq 2^{n}-1$, if $B_{n, i} \notin E_{j}$ we have

$$
\begin{gathered}
\lambda_{n, i} \leq U^{a_{0}-\beta_{n, i}} V^{\gamma_{n, i}} W^{n-a_{0}-\gamma_{n, i}}, \\
\left(Q^{T} B_{n, i} Q\right)_{\alpha, \alpha} \in\left\{\rho_{j, \mathcal{R}+1}, \mathcal{R}+1\right\}, \quad \alpha=1, \ldots, n,
\end{gathered}
$$

and

$$
a:=\#\left\{\alpha:\left(Q^{T} B_{n, i} Q\right)_{\alpha, \alpha}=\rho_{j, \mathcal{R}+1}\right\}=\beta_{n, i}+\gamma_{n, i} \leq n-m .
$$

Therefore, by definition of $E_{j, \mathcal{R}+1}^{a}$, we get $B_{n, i} \in E_{j, \mathcal{R}+1}^{a}$. Hence, for all $0 \leq i \leq 2^{n}-1$, we have proved that

$$
B_{n, i} \in \bigcup_{a=0}^{n-m} E_{j, \mathcal{R}+1}^{a} \cup E_{j} .
$$

We define

$$
\nu=\sum_{i=0}^{2^{n}-1} \lambda_{n, i} \delta_{B_{n, i}}
$$

which is a laminate by (26).

In order to estimate $\nu\left(E_{j, \mathcal{R}+1}^{a}\right)$, we observe that for $B_{n, i} \in E_{j, \mathcal{R}+1}^{a}$ we have $\max \left\{0, a_{0}+\right.$ $a-n\} \leq \beta_{n, i} \leq \min \left\{a_{0}, a\right\}$. Therefore

$$
\begin{aligned}
\nu\left(E_{j, \mathcal{R}+1}^{a}\right) & =\sum_{\substack{i: \beta_{n, i}+\gamma_{n, i}=a \\
B_{n, i} \in E_{j, \mathcal{R}+1}^{a}}} \lambda_{n, i}=\sum_{b=\max \left\{0, a_{0}+a-n\right\}} \sum_{\substack{i: \beta_{n, i}=b, \gamma_{n, i}=a-b \\
B_{n, i} \in E_{j, \mathcal{R}+1}^{a, 1}}}^{\min \left\{a_{0}, a\right\}} \lambda_{n, i} \\
& \leq \sum_{b=\max \left\{0, a_{0}+a-n\right\}}^{\min \left\{a_{0}, a\right\}}\left(\begin{array}{c}
a_{0} \\
b
\end{array}\right)\left(\begin{array}{c}
n-a_{0} \\
a-b
\end{array}\right) U^{a_{0}-b} V^{a-b} W^{n-a_{0}-a+b},
\end{aligned}
$$

where we have used that the $B_{n, i}\left(0 \leq i \leq 2^{n}-1\right)$ in $E_{j, \mathcal{R}+1}^{a}$ are all different, as well as estimate (32). This concludes the proof. 
The following result constructs a function whose gradient approximates the laminate of the previous lemma and have the desired integrability.

Lemma 7. Let $\alpha \in(0,1)$ and $\delta>0$. Then is a $j_{1} \in \mathbb{N}$ such that for any $j \geq j_{1}$, any bounded open set $\omega \subset \mathbb{R}^{n}$ and any $F \in \Gamma_{+}$such that $\operatorname{dist}\left(F, \bigcup_{a=0}^{n-m} E_{j,|F|}^{a}\right)<r_{j,|F|}$, there exists a piecewise affine homeomorphism $f \in W^{1,1}(\omega, F \omega) \cap C^{\alpha}(\bar{\omega}, \overline{F \omega})$ such that

i) $f(x)=F x$ for all $x \in \partial \omega$,

ii) $\|f-F\|_{C^{\alpha}(\bar{\omega})}<\delta$ and $\left\|f^{-1}-F^{-1}\right\|_{C^{\alpha}(\overline{F \omega})}<\delta$,

iii) $D f(x) \in E_{j}$ a.e. $x \in \omega$,

iv) for all $t>0$,

$$
\frac{|\{x \in \omega:|D f(x)|>t\}|}{|\omega|} \lesssim|F|^{m} t^{-m}
$$

Proof. Let $\mathcal{R}=|F|, a_{0} \in\{0, \ldots, n-m\}, Q \in S O(n)$ and

$$
A=Q \operatorname{diag}(\underbrace{\rho_{j, \mathcal{R}}, \ldots, \rho_{j, \mathcal{R}}}_{a_{0}}, \underbrace{\mathcal{R}, \ldots, \mathcal{R}}_{n-a_{0}}) Q^{T} \in E_{j, \mathcal{R}}^{a_{0}}
$$

be such that $|F-A|<r_{j, \mathcal{R}}$, and for $a=0, \ldots, n-m$, define the sets

$$
S_{j, \mathcal{R}}^{a}:=\left\{M \in \Gamma_{+}: \operatorname{dist}\left(M, E_{j, \mathcal{R}}^{a}\right)<r_{j, \mathcal{R}}\right\} .
$$

Note that the sets $S_{j, \mathcal{R}}^{0}, \ldots, S_{j, \mathcal{R}}^{n-m}$ are pairwise disjoint. Indeed, if $S_{j, \mathcal{R}}^{a_{1}} \cap S_{j, \mathcal{R}}^{a_{2}} \neq \emptyset$ for some $a_{1} \neq a_{2}$ we would obtain, thanks to inequality (23), $\left|\mathcal{R}-\rho_{j, \mathcal{R}}\right|<2 r_{j, \mathcal{R}}$, which contradicts the definition of $r_{j, \mathcal{R}}$.

Given $k \in \mathbb{N}$, we define $\tilde{k}=k+\mathcal{R}-1$. We will construct by induction a sequence $\left\{f_{k}\right\}_{k \in \mathbb{N}}$ of piecewise affine homeomorphisms such that

(a) $f_{k}(x)=F x$ for all $x \in \partial \omega$.

(b) $\left\|f_{k}-f_{k-1}\right\|_{C^{\alpha}(\bar{\omega})}<2^{-k} \delta$ and $\left\|f_{k}^{-1}-f_{k-1}^{-1}\right\|_{C^{\alpha}(\overline{F \omega})}<2^{-k} \delta$.

(c) $D f_{k}(x) \in E_{j} \cup \bigcup_{a=0}^{n-m} S_{j, \tilde{k}}^{a}$ for a.e. $x \in \omega$.

(d) $\left|D f_{k}\right|<\tilde{k}+1$ in $\omega \backslash \omega_{k}$, with $\omega_{k}:=\bigcup_{a=0}^{n-m} \omega_{k}^{a}$ and

$\omega_{k}^{a}:=\left\{x \in \omega: f_{k}\right.$ is affine in a neighbourhood of $x$ and $\left.D f_{k}(x) \in S_{j, \tilde{k}}^{a}\right\}, \quad 0 \leq a \leq n-m$.

(e) There exists $j_{1} \in \mathbb{N}$ such that for any $j \geq j_{1}$ we have

$$
\frac{\left|\omega_{k}^{a}\right|}{|\omega|} \lesssim \tilde{k}^{\frac{1}{2}+a-n} \quad \text { for } 0 \leq a \leq n-m-1, \quad \text { and } \quad \frac{\left|\omega_{k}^{n-m}\right|}{|\omega|} \lesssim \tilde{k}^{-m} \sum_{d=1}^{k} \tilde{d}^{-\frac{4}{3}}
$$


(f) $\omega_{k} \supset \omega_{k+1}$ and $\left.f_{k+1}\right|_{\omega \backslash \omega_{k}}=\left.f_{k}\right|_{\omega \backslash \omega_{k}}$.

Note that the sets $\omega_{k}^{a}$ defined in (dd) are open and pairwise disjoint, since so are $S_{j, \mathcal{R}}^{a}$. Recall also that $\tilde{d}$ stands for $d+\mathcal{R}-1$.

For $k=0,1$ we see that the choices $f_{0}(x)=f_{1}(x)=F x, \omega_{0}^{a_{0}}=\omega_{1}^{a_{0}}=\omega$ and $\omega_{0}^{a}=\omega_{1}^{a}=\emptyset$ for $a \neq a_{0}$ satisfy all the assumptions.

Fix $k \in \mathbb{N}$ and assume $f_{k}$ has been constructed. We obtain $f_{k+1}$ by modifying $f_{k}$ on the sets $\omega_{k}^{a}$. Since $f_{k}$ is piecewise affine, there exists a family $\left\{\omega_{i}\right\}_{i \in \mathbb{N}} \subset \omega$ of pairwise disjoint open sets such that $\left|\omega \backslash \bigcup_{i \in \mathbb{N}} \omega_{i}\right|=0$ and $\left.f\right|_{\omega_{i}}$ is affine for each $i \in \mathbb{N}$. More precisely, fix $a \in\{0, \ldots, n-m\}$ and define $\omega_{k, i}^{a}:=\omega_{i} \cap \omega_{k}^{a}$ for each $i \in \mathbb{N}$, which is an open set. From now on, we only deal with those $\omega_{k, i}^{a}$ that are non-empty. Then there exist families $\left\{A_{k, i}^{a}\right\}_{i \in \mathbb{N}} \subset S_{j, \tilde{k}}^{a}$ and $\left\{b_{k, i}^{a}\right\}_{i \in \mathbb{N}} \subset \mathbb{R}^{n}$ such that $f_{k}(x)=A_{k, i}^{a} x+b_{k, i}^{a}$ for $x \in \omega_{k, i}^{a}$.

Let $\nu_{A_{k, i}^{a}}$ be the laminate of Lemma 6 that satisfies $\overline{\nu_{A_{k, i}^{a}}^{a}}=A_{k, i}^{a}$,

$$
\operatorname{supp} \nu_{A_{k, i}^{a}} \subset\left(\bigcup_{b=0}^{n-m} E_{j, \tilde{k}+1}^{b} \cup E_{j}\right) \cap\left\{\xi \in \mathbb{R}^{n \times n}:|\xi| \leq \tilde{k}+1\right\}
$$

and for $0 \leq b \leq n-m$,

$$
\nu_{A_{k, i}^{a}}\left(E_{j, \tilde{k}+1}^{b}\right) \leq C(j, \tilde{k}, a, b) .
$$

We apply Proposition 3 to that laminate and obtain a piecewise affine homeomorphism $g_{k, i}^{a}$ : $\omega_{k, i}^{a} \rightarrow A_{k, i}^{a} \omega_{k, i}^{a}+b_{k, i}^{a}$ with

(g) $g_{k, i}^{a}(x)=A_{k, i}^{a} x+b_{k, i}^{a}$ on $\partial \omega_{k, i}^{a}$.

(h) $\left|D g_{k, i}^{a}(x)\right|<\tilde{k}+2$ a.e. in $\omega_{k, i}^{a}$.

(i) $\left\|g_{k, i}^{a}-f_{k}\right\|_{C^{\alpha}\left(\overline{\omega_{k, i}^{a}}\right)}<2^{-k-2} \delta$ and $\left\|\left(g_{k, i}^{a}\right)^{-1}-f_{k}^{-1}\right\|_{C^{\alpha}\left(A_{k, i}^{a} \overline{\omega_{k, i}^{a}}+b_{k, i}^{a}\right)}<2^{-k-2} \delta$.

(j) $D g_{k, i}^{a}(x) \in E_{j} \cup \bigcup_{b=0}^{n-m} S_{j, \tilde{k}+1}^{b}$ a.e. in $\omega_{k, i}^{a}$.

(k) $\left|\left\{x \in \omega_{k, i}^{a}: D g_{k, i}^{a}(x) \in S_{j, \tilde{k}+1}^{b}\right\}\right| \leq C(j, \tilde{k}, a, b)\left|\omega_{k, i}^{a}\right|$.

In property (j]) we have used that $E_{j}$ is open. We define the piecewise affine function

$$
f_{k+1}(x)= \begin{cases}f_{k}(x) & \text { if } x \in \bar{\omega} \backslash \bigcup_{i=1}^{\infty} \bigcup_{a=0}^{n-m} \omega_{k, i}^{a}, \\ g_{k, i}^{a}(x) & \text { if } x \in \omega_{k, i}^{a} \text { for some } i \in \mathbb{N} \text { and } a \in\{0, \ldots, n-m\},\end{cases}
$$

which is a homeomorphism due to Lemma 5. Property (a) holds for $k+1$ since $f_{k+1}=f_{k}$ on $\partial \omega$. Property (b) holds for $k+1$ thanks to (2i) and Lemma 5. Property (f) for $k+1$ follows easily from the construction. Property (d) for $k+1$ follows from (그) and (ff). Property (CC) for $k+1$ follows from (j) and (f). Finally, we have to prove (e) for $k+1$. 
By definition of $f_{k+1}$, we have that, up to a set of measure zero,

$$
\omega_{k+1}^{b}=\bigcup_{a=0}^{n-m} \bigcup_{i=1}^{\infty}\left\{x \in \omega_{k, i}^{a}: D g_{k, i}^{a}(x) \in S_{j, \tilde{k}+1}^{b}\right\}
$$

with disjoint union, hence for $b=0, \ldots, n-m$,

$$
\begin{aligned}
\frac{\left|\omega_{k+1}^{b}\right|}{|\omega|} & =\sum_{a=0}^{n-m} \sum_{i=1}^{\infty} \frac{\left|\omega_{k, i}^{a}\right|}{|\omega|} \frac{\left|\left\{x \in \omega_{k, i}^{a}: D g_{k, i}^{a}(x) \in S_{j, \tilde{k}+1}^{b}\right\}\right|}{\left|\omega_{k, i}^{a}\right|} \leq \sum_{a=0}^{n-m} \frac{\left|\omega_{k}^{a}\right|}{|\omega|} C(j, \tilde{k}, a, b) \\
& \lesssim \sum_{a=0}^{n-m-1} \tilde{k}^{\frac{1}{2}+a-n} C(j, \tilde{k}, a, b)+C(j, \tilde{k}, n-m, b) \tilde{k}^{-m} \sum_{d=1}^{k} \tilde{d}^{-\frac{4}{3}},
\end{aligned}
$$

where we have used (즈) and (eㅡ). So, in order to prove (ㅌ) for $k+1$ it is enough to show that there exist $j_{0} \in \mathbb{N}$ and $k_{0} \in \mathbb{N}$ such that if $k \geq k_{0}$ and $j \geq j_{0}$ then

$$
\sum_{a=0}^{n-m-1} \tilde{k}^{\frac{1}{2}+a-n} C(j, \tilde{k}, a, b)+C(j, \tilde{k}, n-m, b) \tilde{k}^{-m} \sum_{d=1}^{k} \tilde{d}^{-\frac{4}{3}} \leq(\tilde{k}+1)^{\frac{1}{2}+b-n}
$$

for $0 \leq b \leq n-m-1$, and

$$
\sum_{a=0}^{n-m-1} \tilde{k}^{\frac{1}{2}+a-n} C(j, \tilde{k}, a, n-m)+C(j, \tilde{k}, n-m, n-m) \tilde{k}^{-m} \sum_{d=1}^{k} \tilde{d}^{-\frac{4}{3}} \leq(\tilde{k}+1)^{-m} \sum_{d=1}^{k+1} \tilde{d}^{-\frac{4}{3}} .
$$

Recall from (22) that, for $k \geq 2$,

$$
C(j, \tilde{k}, a, b)=(\tilde{k}+1)^{-n+b} \sum_{\ell=\max \{0, a+b-n\}}^{\min \{a, b\}}\left(\begin{array}{l}
a \\
\ell
\end{array}\right)\left(\begin{array}{c}
n-a \\
b-\ell
\end{array}\right)\left(\tilde{k}+r_{j, \tilde{k}}\right)^{n-a-b+\ell} \tilde{k}^{m(\ell-a)+a-b} 2^{-j(a-\ell)} .
$$

Using the inequality $\left(\tilde{k}+r_{j, \tilde{k}}\right)^{n-a-b+\ell} \leq \tilde{k}^{n-a-b+\ell}+2^{n} \tilde{k}^{n-a-b+\ell-1} r_{j, \tilde{k}}$, we find that

$$
\begin{aligned}
C(j, \tilde{k}, a, b) & \leq \\
& (\tilde{k}+1)^{b-n} \sum_{\ell=\max \{0, a+b-n\}}^{\min \{a, b\}}\left(\begin{array}{l}
a \\
\ell
\end{array}\right)\left(\begin{array}{c}
n-a \\
b-\ell
\end{array}\right) \tilde{k}^{n+m(\ell-a)+\ell-2 b}\left[1+2^{n} r_{j, \tilde{k}^{-1}}\right] 2^{-j(a-\ell)} .
\end{aligned}
$$

We will also use the cruder inequality

$$
C(j, \tilde{k}, a, b) \lesssim(\tilde{k}+1)^{b-n} \tilde{k}^{n+\min \{a, b\}(m+1)-a m-2 b} .
$$

In order to show inequalities (33) and (34), we estimate $C(j, \tilde{k}, a, b)$ according to whether $a$ or $b$ equal $n-m$ or are less than it. We first observe that for $a, b \in\{0, \ldots, n-m\}$ and $\ell \in\{0, \ldots, \min \{a, b\}\}$ we have

$$
\begin{cases}m(\ell-a)+\ell+a-2 b=0 & \text { if } a=b=\ell \\ m(\ell-a)+\ell+a-2 b \leq-1 & \text { otherwise. }\end{cases}
$$


In the case $0 \leq b \leq n-m-1$ we have that exists a constant $c_{1}(n)$ depending on $n$ such that, for $k \geq 2$,

$$
\begin{aligned}
& (\tilde{k}+1)^{n-b} \sum_{a=0}^{n-m-1} \tilde{k}^{\frac{1}{2}+a-n} C(j, \tilde{k}, a, b) \\
& \leq \sum_{a=0}^{n-m-1} \sum_{\ell=\max \{0, a+b-n\}}^{\min \{a, b\}}\left(\begin{array}{l}
a \\
\ell
\end{array}\right)\left(\begin{array}{c}
n-a \\
b-\ell
\end{array}\right) \tilde{k}^{m(\ell-a)+\ell+a-2 b}\left[\tilde{k}^{\frac{1}{2}}+2^{n} r_{j, \tilde{k}} \tilde{k}^{-\frac{1}{2}}\right] 2^{-j(a-\ell)} \\
& \leq \tilde{k}^{\frac{1}{2}}+c_{1}(n) \frac{2^{-j}}{\tilde{k}^{\frac{1}{2}}}
\end{aligned}
$$

SO

$$
(\tilde{k}+1)^{n-\frac{1}{2}-b} \sum_{a=0}^{n-m-1} \tilde{k}^{\frac{1}{2}+a-n} C(j, \tilde{k}, a, b) \leq\left(\frac{\tilde{k}}{\tilde{k}+1}\right)^{\frac{1}{2}}+c_{1}(n) \frac{2^{-j}}{\tilde{k}^{\frac{1}{2}}(\tilde{k}+1)^{\frac{1}{2}}}
$$

whereas

$$
C(j, \tilde{k}, n-m, b) \tilde{k}^{-m}(\tilde{k}+1)^{n-b} \lesssim \tilde{k}^{n+b(m+1)-(n-m) m-2 b-m} \leq \tilde{k}^{1-m} \leq \tilde{k}^{-1}
$$

So

$$
C(j, \tilde{k}, n-m, b) \tilde{k}^{-m} \sum_{d=1}^{k} \tilde{d}^{-\frac{4}{3}}(\tilde{k}+1)^{n-\frac{1}{2}-b} \leq c_{1}(n) \tilde{k}^{-\frac{3}{2}} .
$$

Given the previous constant $c_{1}(n)$, let $j_{1} \in \mathbb{N}$ be such that for all $j \geq j_{1}$ and $k \geq 2$,

$$
\left(\frac{\tilde{k}}{\tilde{k}+1}\right)^{\frac{1}{2}}+c_{1}(n)\left(\frac{2^{-j}}{\tilde{k}^{\frac{1}{2}}(\tilde{k}+1)^{\frac{1}{2}}}+\tilde{k}^{-\frac{3}{2}}\right) \leq 1 .
$$

Then using (35), (36) and (37)

$$
\begin{aligned}
& (\tilde{k}+1)^{n-\frac{1}{2}-b}\left[\sum_{a=0}^{n-m-1} \tilde{k}^{\frac{1}{2}+a-n} C(j, \tilde{k}, a, b)+C(j, \tilde{k}, n-m, b) \tilde{k}^{-m} \sum_{d=1}^{k} \tilde{d}^{-\frac{4}{3}}\right] \\
& \leq\left(\frac{\tilde{k}}{\tilde{k}+1}\right)^{\frac{1}{2}}+c_{1}(n)\left(\frac{2^{-j}}{\tilde{k}^{\frac{1}{2}}(\tilde{k}+1)^{\frac{1}{2}}}+\tilde{k}^{-\frac{3}{2}}\right) \leq 1,
\end{aligned}
$$

which proves (33). In the case $b=n-m$,

$$
(\tilde{k}+1)^{m} C(j, \tilde{k}, a, n-m) \lesssim \tilde{k}^{2 m+a-n}
$$

so

$$
\sum_{a=0}^{n-m-1} \tilde{k}^{\frac{1}{2}+a-n} C(j, \tilde{k}, a, n-m)(\tilde{k}+1)^{m} \lesssim \sum_{a=0}^{n-m-1} \tilde{k}^{\frac{1}{2}+2(m+a-n)} \lesssim \tilde{k}^{-\frac{3}{2}}
$$


and, hence, there exists a constant $c_{2}(n)$ such that

$$
\sum_{a=0}^{n-m-1} \tilde{k}^{\frac{1}{2}+a-n} C(j, \tilde{k}, a, n-m)(\tilde{k}+1)^{m} \leq c_{2}(n) \tilde{k}^{-\frac{3}{2}} .
$$

Recall that

$$
2^{n} r_{j, \tilde{k}} \leq \frac{1}{2}(\tilde{k}+1)^{-m-1}
$$

Then, splitting the following sum in the case $\ell=n-m$ and the case $\ell<n-m$, we have

$$
\begin{aligned}
& C(j, \tilde{k}, n-m, n-m) \tilde{k}^{-m}(\tilde{k}+1)^{m} \\
& \leq \sum_{\ell=\max \{0, n-2 m\}}^{n-m}\left(\begin{array}{c}
n-m \\
\ell
\end{array}\right)\left(\begin{array}{c}
m \\
n-m-\ell
\end{array}\right) \tilde{k}^{-n+m(\ell-n+m+1)+\ell}\left[1+2^{n} r_{j, \tilde{k}} \tilde{k}^{-1}\right] \\
& \leq 1+c_{2}(n) \tilde{k}^{-m-1} .
\end{aligned}
$$

In addition, there exists $k_{0} \in \mathbb{N}$ such that for $k \geq k_{0}$

$$
c_{2}(n)\left(\tilde{k}^{-\frac{3}{2}}+\tilde{k}^{-m-1} \sum_{d=1}^{\infty} \tilde{d}^{-\frac{4}{3}}\right) \leq(\tilde{k}+1)^{-\frac{4}{3}} .
$$

Therefore, for $j \in \mathbb{N}$ and $k \geq k_{0}$ we use (38), (39) and (40) to get

$$
\begin{aligned}
& (\tilde{k}+1)^{m}\left[\sum_{a=0}^{n-m-1} \tilde{k}^{\frac{1}{2}+a-n} C(j, \tilde{k}, a, n-m)+C(j, \tilde{k}, n-m, n-m) \tilde{k}^{-m} \sum_{d=1}^{k} \tilde{d}^{-\frac{4}{3}}\right] \\
& \leq c_{2}(n) \tilde{k}^{-\frac{3}{2}}+\sum_{d=1}^{k} \tilde{d}^{-\frac{4}{3}}\left(1+c_{2}(n) \tilde{k}^{-m-1}\right) \leq \sum_{d=1}^{k+1} \tilde{d}^{-\frac{4}{3}} .
\end{aligned}
$$

This proves (34) and the construction of $\left\{f_{k}\right\}_{k \in \mathbb{N}}$ is finished.

From (e्e) we obtain

$$
\frac{\left|\omega_{k}\right|}{|\omega|}=\sum_{a=0}^{n-m} \frac{\left|\omega_{k}^{a}\right|}{|\omega|} \lesssim \sum_{a=0}^{n-m-1} \tilde{k}^{\frac{1}{2}+a-n}+\tilde{k}^{-m} \sum_{d=1}^{k} \tilde{d}^{-\frac{4}{3}} \lesssim \tilde{k}^{-m} .
$$

By (b) , the sequences $\left\{f_{k}\right\}_{k=1}^{\infty}$ and $\left\{f_{k}^{-1}\right\}_{k=1}^{\infty}$ converge in the $C^{\alpha}$ norm. We define $f$ as the limit of $f_{k}$. Thanks to the uniform convergence, the limit of $f_{k}^{-1}$ is the inverse of $f$. Thus, $f$ is a homeomorphism. In addition, $f$ is piecewise affine. To check this, we see from (f) that $f_{k+1}=f_{k} \chi_{\omega \backslash \omega_{k}}+g_{k} \chi_{\omega_{k}}$ for a certain $g_{k}: \omega_{k} \rightarrow \mathbb{R}^{n}$ piecewise affine. Thus, $f_{k+1}=\sum_{i=1}^{k} g_{i} \chi_{\omega_{i} \backslash \omega_{i+1}}$, so $f=\sum_{i=1}^{\infty} g_{i} \chi_{\omega_{i} \backslash \omega_{i+1}}$, which shows that $f$ is piecewise affine. Moreover, $D f_{k+1}=\sum_{i=1}^{k} D g_{i} \chi_{\omega_{i} \backslash \omega_{i+1}}$, so for any $p \in(1, m)$, thanks to (hin) and (41),

$$
\begin{aligned}
& \int_{\omega}\left|D f_{k+1}\right|^{p} \leq \sum_{i=1}^{k}(\tilde{i}+2)^{p}\left(\left|\omega_{i}\right|-\left|\omega_{i+1}\right|\right) \lesssim \sum_{i=1}^{k} i^{p}\left(\left|\omega_{i}\right|-\left|\omega_{i+1}\right|\right) \\
& =\sum_{i=1}^{k}\left[i^{p}-(i-1)^{p}\right]\left|\omega_{i}\right|-k^{p}\left|\omega_{k+1}\right| \lesssim \sum_{i=1}^{k} i^{p-1}\left|\omega_{i}\right| \lesssim \sum_{i=1}^{k} i^{p-1-m} \lesssim 1,
\end{aligned}
$$


which shows that $f \in W^{1, p}\left(\omega, \mathbb{R}^{n}\right)$.

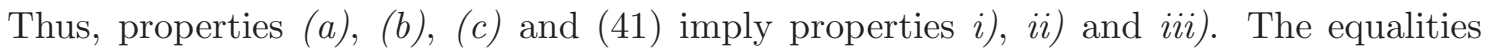
$f(\omega)=F \omega$ and $f(\bar{\omega})=\overline{F \omega}$ are a consequence of $[$ i).

Finally, we estimate the integrability of $D f$. Given $t>0$, let $k_{1}=\max \{1,\lfloor t-\mathcal{R}\rfloor\}$. By (d) and (f), we obtain that for all $k \geq k_{1}$,

$$
\left|D f_{k}\right| \leq \tilde{k}_{1}+1 \quad \text { in } \omega \backslash \omega_{k_{1}}
$$

so

$$
|D f| \leq \tilde{k}_{1}+1 \quad \text { in } \omega \backslash \omega_{k_{1}}
$$

Therefore,

$$
\{x \in \omega:|D f(x)|>t\} \subset \omega_{k_{1}},
$$

hence, from (41), we obtain

$$
\frac{|\{x \in \omega:|D f(x)|>t\}|}{|\omega|} \lesssim \max \left\{1, t^{-m}\right\}
$$

Since $\operatorname{dist}\left(F, \bigcup_{a=0}^{n-m} E_{j,|F|}^{a}\right)<r_{j,|F|}$ we have $|F|>\frac{1}{2}$; therefore iv) follows.

Next, we construct a laminate that goes from $E_{j}$ to $E_{j+m}$. Again, its proof follows the construction of Section 2 ,

Lemma 8. Let $j \in \mathbb{N}$ and $A \in E_{j}$. Then there exist $N \in \mathbb{N} \cap\left[2,2^{n}\right]$,

$$
P_{1} \in E_{j+m} ; \quad P_{i} \in E_{j+m} \cup \bigcup_{a=0}^{n-m} E_{j+m}^{a}, \quad 2 \leq i \leq N ; \quad \lambda_{i} \in[0,1], \quad 1 \leq i \leq N
$$

such that $\nu_{A}:=\sum_{i=1}^{N} \lambda_{i} \delta_{P_{i}}$ belongs to $\mathcal{L}\left(\mathbb{R}^{n \times n}\right), \overline{\nu_{A}}=A, P_{i} \neq P_{j}$ for $i \neq j$,

$$
\left|A-P_{1}\right|<2^{-j} ; \quad\left|P_{i}\right|=|A|, \quad 1 \leq i \leq N ; \quad 1-\lambda_{1} \lesssim \frac{2^{-j}}{|A| \max \left\{1,|A|^{m-1}\right\}}
$$

Proof. Since $A \in E_{j}$, there exist $\sigma_{1} \leq \cdots \leq \sigma_{n}$ and $Q \in S O(n)$ such that $2^{-j-m}<$ $\sigma_{i} \max \left\{1, \sigma_{n}^{m-1}\right\}<2^{-j}$ for $1 \leq i \leq n-m+1$, and $A=Q \operatorname{diag}\left(\sigma_{1}, \ldots, \sigma_{n}\right) Q^{T}$.

Since $\rho_{j+m, \sigma_{n}}=\frac{3 \cdot 2^{-j-2-m}}{\max \left\{1, \sigma_{n}^{m-1}\right\}}$, we have $\rho_{j+m, \sigma_{n}}<\sigma_{1}$. As in Lemma 6, we shall construct families (25) by finite induction on $\ell$. Let $B_{0,0}=A, \lambda_{0,0}=1$ and for $0 \leq \ell \leq n-1$, $0 \leq i \leq 2^{\ell}-1$, we assume $\left\{B_{\ell, i}\right\}_{i=0}^{2^{\ell}-1}$ and $\left\{\lambda_{\ell, i}\right\}_{i=0}^{2^{\ell}-1}$ have been defined, $Q^{T} B_{\ell, j} Q$ is diagonal, $\lambda_{\ell, i} \geq 0$, equations (26) and (27) hold, $\left|B_{\ell, i}\right|=\sigma_{n}$,

$$
B_{\ell, 0}=Q \operatorname{diag}(\underbrace{\rho_{j+m, \sigma_{n}}, \ldots, \rho_{j+m, \sigma_{n}}}_{\min \{\ell, n-m+1\}}, \sigma_{\min \{\ell, n-m+1\}+1}, \ldots, \sigma_{n}) Q^{T},
$$


and

$$
\lambda_{\ell, 0}=\prod_{k=1}^{\min \{\ell, n-m+1\}} \frac{\sigma_{n}-\sigma_{k}}{\sigma_{n}-\rho_{j+m, \sigma_{n}}} .
$$

We also assume that if $B_{\ell, i} \notin E_{j+m}$ then

$$
\left(Q^{T} B_{\ell, i} Q\right)_{\alpha, \alpha} \in\left\{\rho_{j+m, \sigma_{n}}, \sigma_{n}\right\}, \quad \alpha=1, \ldots, \ell .
$$

With the above induction hypotheses, we construct $\left\{B_{\ell+1, i}\right\}_{i=0}^{2^{\ell+1}-1}$ and $\left\{\lambda_{\ell+1, i}\right\}_{i=0}^{2^{\ell+1}-1}$ as follows. For any $0 \leq i \leq 2^{\ell}-1$, let

$$
\begin{aligned}
& B_{\ell+1,2 i}= \begin{cases}B_{\ell, i}-Q \operatorname{diag}(\underbrace{0, \ldots, 0,}_{\ell} \sigma_{\ell+1}-\rho_{j+m, \sigma_{n}}, \underbrace{0, \ldots, 0}_{n-\ell-1}) Q^{T} & \text { if } B_{\ell, i} \notin E_{j+m}, \\
B_{\ell, i} & \text { if } B_{\ell, i} \in E_{j+m},\end{cases} \\
& B_{\ell+1,2 i+1}= \begin{cases}B_{\ell, i}-Q \operatorname{diag}(\underbrace{0, \ldots, 0,}_{\ell} \sigma_{\ell+1}-\sigma_{n}, \underbrace{0, \ldots, 0}_{n-\ell-1}) Q^{T} & \text { if } B_{\ell, i} \notin E_{j+m}, \\
B_{\ell, i} & \text { if } B_{\ell, i} \in E_{j+m},\end{cases} \\
& \lambda_{\ell+1,2 i}= \begin{cases}\frac{\sigma_{n}-\sigma_{\ell+1}}{\sigma_{n}-\rho_{j+m, \sigma_{n}}} \lambda_{\ell, i} & \text { if } B_{\ell, i} \notin E_{j+m}, \\
\lambda_{\ell, i} & \text { if } B_{\ell, i} \in E_{j+m},\end{cases} \\
& \lambda_{\ell+1,2 i+1}= \begin{cases}\frac{\sigma_{\ell+1}-\rho_{j+m, \sigma_{n}}}{\sigma_{n}-\rho_{j+m, \sigma_{n}}} \lambda_{\ell, i} & \text { if } B_{\ell, i} \notin E_{j+m}, \\
0 & \text { if } B_{\ell, i} \in E_{j+m} .\end{cases}
\end{aligned}
$$

Hence

$$
B_{\ell, i}=\frac{\sigma_{n}-\sigma_{\ell+1}}{\sigma_{n}-\rho_{j+m, \sigma_{n}}} B_{\ell+1,2 i}+\frac{\sigma_{\ell+1}-\rho_{j+m, \sigma_{n}}}{\sigma_{n}-\rho_{j+m, \sigma_{n}}} B_{\ell+1,2 i+1} .
$$

Using that $2^{-j-2 m}<\rho_{j+m, \sigma_{n}} \sigma_{n}<2^{-j-m}$ and the definition of $E_{j}$, we have that $B_{\ell, 0} \in E_{j+m}$ if and only if $\ell \geq n-m+1$. Hence, if $B_{\ell, 0} \in E_{j+m}$,

$$
\lambda_{\ell+1,0}=\lambda_{\ell, 0}=\prod_{k=1}^{n-m+1} \frac{\sigma_{n}-\sigma_{k}}{\sigma_{n}-\rho_{j+m, \sigma_{n}}}=\prod_{k=1}^{\min \{\ell+1, n-m+1\}} \frac{\sigma_{n}-\sigma_{k}}{\sigma_{n}-\rho_{j+m, \sigma_{n}}},
$$

whereas if $B_{\ell, 0} \notin E_{j+m}$,

$$
\lambda_{\ell+1,0}=\frac{\sigma_{n}-\sigma_{\ell+1}}{\sigma_{n}-\rho_{j+m, \sigma_{n}}} \lambda_{\ell, i}=\prod_{k=1}^{\ell+1} \frac{\sigma_{n}-\sigma_{k}}{\sigma_{n}-\rho_{j+m, \sigma_{n}}}=\prod_{k=1}^{\min \{\ell+1, n-m+1\}} \frac{\sigma_{n}-\sigma_{k}}{\sigma_{n}-\rho_{j+m, \sigma_{n}}} .
$$

With this, it is clear that $\left\{B_{\ell+1, i}\right\}_{i=0}^{2^{\ell+1}-1}$ and $\left\{\lambda_{\ell+1, i}\right\}_{i=0}^{2^{\ell+1}-1}$ satisfy the induction hypotheses. 
For $i=1, \ldots, 2^{n}$ define $\hat{\lambda}_{i}=\lambda_{n, i-1}$ and $\hat{P}_{i}=B_{n, i-1}$. In order to make the $\hat{P}_{i}$ distinct, we let $N \in \mathbb{N}$ and $P_{1}, \ldots, P_{N}$ be such that

$$
\left\{P_{1}, \ldots, P_{N}\right\}=\left\{\hat{P}_{1}, \ldots, \hat{P}_{2^{n}}\right\}, \quad P_{i} \neq P_{j} \text { if } i \neq j, \quad P_{1}=\hat{P}_{1}
$$

and define

$$
\lambda_{i}=\sum_{j: \hat{P}_{j}=P_{i}} \hat{\lambda}_{j}, \quad i=1, \ldots, N
$$

We have shown that $\nu_{A} \in \mathcal{L}\left(\mathbb{R}^{n \times n}\right)$ and $\overline{\nu_{A}}=B_{0,0}=A$. Now we estimate the distance between $A$ and $P_{1}$ :

$$
\begin{aligned}
\left|A-P_{1}\right| & =\left|Q \operatorname{diag}(\underbrace{\sigma_{1}-\rho_{j+m, \sigma_{n}}, \ldots, \sigma_{n-m+1}-\rho_{j+m, \sigma_{n}}}_{n-m+1}, \underbrace{0, \ldots, 0}_{m-1}) Q^{T}\right| \\
& =\sigma_{n-m+1}-\rho_{j+m, \sigma_{n}}<\sigma_{n-m+1}<2^{-j} .
\end{aligned}
$$

To finish the proof it only remains to check the last estimate of (43). Notice that

$$
\lambda_{1} \geq \hat{\lambda}_{1}=\prod_{k=1}^{n-m+1} \frac{\sigma_{n}-\sigma_{k}}{\sigma_{n}-\rho_{j+m, \sigma_{n}}}=\prod_{k=1}^{n-m+1}\left(1-\frac{\sigma_{k}-\rho_{j+m, \sigma_{n}}}{\sigma_{n}-\rho_{j+m, \sigma_{n}}}\right) \geq \prod_{k=1}^{n-m+1}\left(1-\frac{\sigma_{k}}{\sigma_{n}}\right) .
$$

If $|A| \geq 1$, for $1 \leq k \leq n-m+1$ we have $\sigma_{k} \sigma_{n}^{m-1}<2^{-j}$, so

$$
1-\lambda_{1} \leq 1-\prod_{k=1}^{n-m+1}\left(1-\frac{2^{-j}}{\sigma_{n}^{m}}\right)=1-\left(1-\frac{2^{-j}}{\sigma_{n}^{m}}\right)^{n-m+1} \lesssim \frac{2^{-j}}{\sigma_{n}^{m}}=\frac{2^{-j}}{|A|^{m}} .
$$

If, on the other hand, $|A|<1$, then $\sigma_{k}<2^{-j}$ for $1 \leq k \leq n-m+1$, and since $A \in E_{j}$, we know that $|A|>\frac{1}{2}$, so

$$
1-\lambda_{1} \leq 1-\prod_{k=1}^{n-m+1}\left(1-\frac{2^{-j}}{\sigma_{n}}\right)=1-\left(1-\frac{2^{-j}}{\sigma_{n}}\right)^{n-m+1} \lesssim \frac{2^{-j}}{\sigma_{n}}=\frac{2^{-j}}{|A|}
$$

and the proof is finished.

A variant of Lemma 8 will be needed. If, instead of starting from an $A \in E_{j}$, we begin with the identity matrix, the same proof of Lemma 8 yields the following result, which will be used in the first step of the construction of the sequence approximating the final homeomorphism of Theorem 1 .

Lemma 9. Given $\alpha \in(0,1)$ and $\delta>0$, let $j_{1} \in \mathbb{N}$ be as in Lemma 7 . Then there exist $N \in \mathbb{N} \cap\left[2,2^{n}\right]$

$$
P_{1} \in E_{j_{1}} ; \quad P_{i} \in E_{j_{1}} \cup \bigcup_{a=0}^{n-m} E_{j_{1}}^{a}, \quad 2 \leq i \leq N ; \quad \lambda_{i} \in[0,1], \quad 1 \leq i \leq N
$$


such that $\nu_{I}:=\sum_{i=1}^{N} \lambda_{i} \delta_{P_{i}}$ belongs to $\mathcal{L}\left(\mathbb{R}^{n \times n}\right), \overline{\nu_{I}}=I, P_{i} \neq P_{j}$ for $i \neq j$ and

$$
|I| \lesssim\left|P_{i}\right| \lesssim|I|, \quad 1 \leq i \leq N
$$

Next, we approximate the laminate of Lemma 8 by a function.

Lemma 10. Let $A \in E_{j}$. For any bounded open $\omega \subset \mathbb{R}^{n}, \alpha \in(0,1)$ and $\eta>0$ there exists a piecewise affine homeomorphism $h \in W^{1,1}\left(\omega, \mathbb{R}^{n}\right) \cap C^{\alpha}(\bar{\omega})$ satisfying

(a) $h(x)=A x$ on $\partial \omega$.

(b) $\|h-A\|_{C^{\alpha}(\bar{\omega})}<\eta$ and $\left\|h^{-1}-A^{-1}\right\|_{C^{\alpha}(\overline{A \omega})}<\eta$.

(c) $\operatorname{Dh}(x) \in E_{j+m}$ a.e. $x \in \omega$.

(d) $\int_{\omega}|D h(x)-A| d x \lesssim 2^{-j}|\omega|$.

(e) There exists an open set $\tilde{\omega} \subset \omega$ such that

(e1) $|D h(x)-A| \lesssim 2^{-j}$ a.e. $x \in \omega \backslash \tilde{\omega}$.

(라) $|\{x \in \tilde{\omega}:|D h(x)|>t\}| \lesssim 2^{-j}|\omega| t^{-m}$.

Proof. First we build the laminate of Lemma 8

$$
\nu_{A}=\sum_{i=1}^{N} \lambda_{i} \delta_{P_{i}} \in \mathcal{L}\left(\mathbb{R}^{n \times n}\right)
$$

satisfying $\overline{\nu_{A}}=A$, (42) and (43). Let $\varepsilon>0$ be such that

$$
\varepsilon<\min \left\{\frac{1}{2} r_{j,|A|}, 2^{-j}-\left|A-P_{1}\right|, \frac{1}{2} \min _{2 \leq i \leq N}\left|P_{1}-P_{i}\right|\right\}
$$

and

$$
r_{j,|A|}<2 r_{j, \mathcal{R}} \quad \text { for } \mathcal{R} \in(|A|-\varepsilon,|A|+\varepsilon) .
$$

Then, Proposition 3 gives a piecewise affine homeomorphism $g: \omega \rightarrow A \omega$ satisfying

1) $g(x)=A x$ on $\partial \omega$

2) $\|g-A\|_{C^{\alpha}(\bar{\omega})}<\frac{\eta}{2}$ and $\left\|g^{-1}-A^{-1}\right\|_{C^{\alpha}(A \bar{\omega})}<\frac{\eta}{2}$,

3) $\left|\left\{x \in \omega:\left|D g(x)-P_{i}\right|<\varepsilon\right\}\right|=\lambda_{i}|\omega|$ for $i=1, \ldots, N$.

Let $\tilde{\omega}=\left\{x \in \omega: g\right.$ is affine in a neighbourhood of $x$ and $\left.\left|D g(x)-P_{1}\right|>\varepsilon\right\}$ and

$$
\hat{\omega}=\left\{x \in \omega: \begin{array}{l}
g \text { is affine in a neighbourhood of } x \text { and } \\
\left|D g(x)-P_{i}\right|<\varepsilon \text { for some } i \in\{2, \ldots, N\} \text { and } P_{i} \in \bigcup_{a=0}^{n-m} E_{j+m}^{a}
\end{array}\right\} .
$$


As $\operatorname{dist}\left(E_{j+m}, \bigcup_{a=0}^{n-m} E_{j+m}^{a}\right)>\frac{1}{2}$ and $\varepsilon<\frac{1}{4}$, we have that $\hat{\omega} \subset \tilde{\omega}$. Note also that $\hat{\omega}$ and $\tilde{\omega}$ are open. Finally, the choice of $\varepsilon$ was done so that, thanks to 3 ) the set of $x \in \Omega$ such that $\left|D g(x)-P_{1}\right|=\varepsilon$ has measure zero.

Since $g$ is piecewise affine, there exist a family $\left\{\hat{\omega}_{k}\right\}_{k \in \mathbb{N}}$ of open sets such that $\hat{\omega}=\bigcup_{k=1}^{\infty} \hat{\omega}_{k}$, and $\hat{P}_{k} \in \mathbb{R}^{n \times n}, b_{k} \in \mathbb{R}^{n}$ with $g(x)=\hat{P}_{k} x+b_{k}$ in $\hat{\omega}_{k}$. Recalling that $\left|P_{i}\right|=|A|$ for $i=1, \ldots, N$, and ||$A|-| \hat{P}_{k}||<\varepsilon$, we have

$$
\operatorname{dist}\left(\hat{P}_{k}, \bigcup_{a=0}^{n-m} E_{j+m}^{a}\right)<\varepsilon<\frac{1}{2} r_{j,|A|}<r_{j,\left|\hat{P}_{k}\right|}, \quad k \in \mathbb{N} .
$$

We define $h$ as the piecewise affine homeomorphism given by Lemma 7 in each $\hat{\omega}_{k}$ and as $g$ in $\bar{\omega} \backslash \bigcup_{k=1}^{\infty} \hat{\omega}_{k}$. By Lemma [5, $h$ is a homeomorphism, and satisfies (回), (b) and (e1). Property (ㄷ) comes from (iii) in Lemma 7. By (iv) of the same lemma we have

$$
\frac{\left|\left\{x \in \hat{\omega}_{k}:|D h(x)|>t\right\}\right|}{\left|\hat{\omega}_{k}\right|} \lesssim\left|\hat{P}_{k}\right|^{m} t^{-m}, \quad t>0 .
$$

Therefore, using (43) and that for all $k$ there exists $i$ such that $\left|\hat{P}_{k}-P_{i}\right|<\varepsilon$, we have

$$
\frac{|\{x \in \hat{\omega}:|D h(x)|>t\}|}{|\hat{\omega}|} \lesssim|A|^{m} t^{-m}
$$

Now, by (43) and 3), we have

$$
|| D h|-| A|| \leq\left|D h-P_{i}\right|=\left|D g-P_{i}\right|<\varepsilon \quad \text { a.e. in } \omega \backslash \hat{\omega},
$$

for some $i \in\{1, \ldots, N\}$ depending on the point. Hence $|D h|<|A|+\varepsilon$ a.e. in $\tilde{\omega} \backslash \hat{\omega}$. Thus, if $t>|A|+\varepsilon$ we have

$$
|\{x \in \tilde{\omega}:|D h(x)|>t\}|=|\{x \in \hat{\omega}:|D h(x)|>t\}| \lesssim|A|^{m} t^{-m}|\hat{\omega}| \leq|A|^{m} t^{-m}|\tilde{\omega}|,
$$

whereas, if $0<t \leq|A|+\varepsilon$ we get $1 \lesssim|A|^{m} t^{-m}$ and

$$
\begin{aligned}
|\{x \in \tilde{\omega}:|D h(x)|>t\}| & =|\{x \in \tilde{\omega} \backslash \hat{\omega}:|D h(x)|>t\}|+|\{x \in \hat{\omega}:|D h(x)|>t\}| \\
& \lesssim|\tilde{\omega} \backslash \hat{\omega}|+|A|^{m} t^{-m}|\hat{\omega}| \lesssim|A|^{m} t^{-m}|\tilde{\omega}| .
\end{aligned}
$$

Hence, for all $t>0$,

$$
|\{x \in \tilde{\omega}:|D h(x)|>t\}| \lesssim|A|^{m} t^{-m}|\tilde{\omega}| .
$$

So using (44) and

$$
|\tilde{\omega}|=\left(1-\lambda_{1}\right)|\omega| \lesssim \frac{2^{-j}}{|A| \max \left\{1,|A|^{m-1}\right\}}|\omega|,
$$

we have $|\{x \in \tilde{\omega}:|D h(x)|>t\}| \lesssim 2^{-j} t^{-m}|\omega|$, that is, (eq). 
Finally,

$$
\int_{\omega}|D h(x)-A| d x \leq \int_{\tilde{\omega}}|D h(x)| d x+|A||\tilde{\omega}|+\int_{\omega \backslash \tilde{\omega}}|D h(x)-A| d x .
$$

Using now the common formula for calculating the $L^{1}$ norm of a function in terms of its distribution function, as well as (44), we obtain

$$
\begin{aligned}
\int_{\tilde{\omega}}|D h(x)| d x & =\left[\int_{0}^{2|A|}+\int_{2|A|}^{\infty}\right]|\{x \in \tilde{\omega}:|D h(x)|>t\}| d t \\
& \lesssim|A||\tilde{\omega}|+|A|^{m}|\tilde{\omega}| \int_{2|A|}^{\infty} t^{-m} d t \lesssim|A||\tilde{\omega}|,
\end{aligned}
$$

hence, thanks to (45),

$$
\int_{\tilde{\omega}}|D h(x)| d x+|A||\tilde{\omega}| \lesssim|A||\tilde{\omega}| \leq 2^{-j}|\omega|
$$

while (e1) yields

$$
\int_{\omega \backslash \tilde{\omega}}|D h(x)-A| d x \lesssim 2^{-j}|\omega \backslash \tilde{\omega}| \leq 2^{-j}|\omega|
$$

Inequalities (46), (47) and (48) show (di) and finish the proof.

The next lemma is the analogous of the previous one when one starts with the identity matrix. Its proof is similar to that of Lemma 10, but using Lemma 9 instead of Lemma 8 ,

Lemma 11. For any $\alpha \in(0,1)$ and $\eta>0$ there exist $j_{1} \in \mathbb{N}$ and a piecewise affine homeomorphism $h \in W^{1,1}\left(\Omega, \mathbb{R}^{n}\right) \cap C^{\alpha}\left(\bar{\Omega}, \mathbb{R}^{n}\right)$ satisfying

(a) $h(x)=x$ on $\partial \Omega$.

(b) $\|h-I\|_{C^{\alpha}(\bar{\Omega})}<\eta$ and $\left\|h^{-1}-I\right\|_{C^{\alpha}(\bar{\Omega})}<\eta$.

(c) $\operatorname{Dh}(x) \in E_{j_{1}}$ a.e. $x \in \Omega$.

(d) $\int_{\Omega}|D h(x)-I| d x \lesssim \frac{1}{2}$

(e) $|\{x \in \Omega:|D h(x)|>t\}| \lesssim|\Omega| t^{-m}$ for all $t>0$.

\section{Proof of the theorem}

We are in a position to prove Theorem 11 using Lemmas 10 and 11 .

Proof of Theorem 1, Let $j_{1} \in \mathbb{N}$ be as in Lemma 7 . For each $j \in \mathbb{N}$ we will construct a piecewise affine homeomorphism $f_{j} \in W^{1,1}\left(\Omega, \mathbb{R}^{n}\right) \cap C^{\alpha}\left(\bar{\Omega}, \mathbb{R}^{n}\right)$ such that 
1) $f_{j}(x)=x$ on $\partial \Omega$.

2) $\left\|f_{j}-f_{j-1}\right\|_{C^{\alpha}(\bar{\Omega})}<2^{-j} \delta$ and $\left\|f_{j}^{-1}-f_{j-1}^{-1}\right\|_{C^{\alpha}(\bar{\Omega})}<2^{-j} \delta$.

3) $D f_{j} \in E_{j_{1}+(j-1) m}$ a.e.

4) $\int_{\Omega}\left|D f_{j}(x)-D f_{j-1}(x)\right| d x \lesssim 2^{-j}|\Omega|$.

5) $\left|\left\{x \in \Omega:\left|D f_{j}(x)\right|>t\right\}\right| \lesssim|\Omega| t^{-m} \prod_{k=0}^{j-1}\left(1-2^{-k-1}\right)^{-m}\left(1+2^{-k}\right)$.

The construction of $f_{j}$ proceeds by induction. Let $f_{0}=\mathrm{id}$, which does not satisfy (3). We use Lemma 11 to create a piecewise affine homeomorphism $f_{1}$ such that properties 1 15 ) hold for $j=1$.

Now suppose we have $f_{j}$. Since $f_{j}$ is piecewise affine, for each $i \in \mathbb{N}$ there exist $A_{i j} \in \mathbb{R}^{n \times n}$, $b_{i j} \in \mathbb{R}^{n}$ and $\Omega_{i j} \subset \Omega$ open such that $\left|\Omega \backslash \bigcup_{i} \Omega_{i j}\right|=0$ and

$$
f_{j}(x)=A_{i j} x+b_{i j}, \quad x \in \Omega_{i j}
$$

and, by 3), $A_{i j} \in E_{j_{1}+(j-1) m}$. On each $\Omega_{i j}$ we apply Lemma 10, there exists a piecewise affine homeomorphism $h_{i j}: \bar{\Omega}_{i j} \rightarrow A_{i j} \bar{\Omega}_{i j}+b_{i j}$ in $W^{1,1}$ and in $C^{\alpha}$ such that

- $h_{i j}(x)=f_{j}(x)$ for $x \in \partial \Omega_{i j}$.

- $\left\|h_{i j}-f_{j}\right\|_{C^{\alpha}\left(\overline{\Omega_{i j}}\right)}<2^{-(j+2)} \delta$ and $\left\|h_{i j}^{-1}-f_{j}^{-1}\right\|_{C^{\alpha}\left(\bar{\Omega}_{i j}+b_{i j}\right)}<2^{-(j+2)} \delta$.

- $D h_{i j}(x) \in E_{j_{1}+j m}$ a.e. $x \in \Omega_{i j}$.

- $\int_{\Omega_{i j}}\left|D h_{i j}(x)-A_{i j}\right| d x \lesssim 2^{-j}\left|\Omega_{i j}\right|$.

- There exists an open set $\tilde{\Omega}_{i j} \subset \Omega_{i j}$ such that

$$
\left|D h_{i j}(x)-A_{i j}\right| \lesssim 2^{-j} \text { a.e. } x \in \Omega_{i j} \backslash \tilde{\Omega}_{i j} \quad \text { and } \quad \frac{\left|\left\{x \in \tilde{\Omega}_{i j}:\left|D h_{i j}(x)\right|>t\right\}\right|}{\left|\Omega_{i j}\right|} \lesssim 2^{-j} t^{-m}
$$

We define the piecewise affine function $f_{j+1}: \bar{\Omega} \rightarrow \mathbb{R}^{n}$ as

$$
f_{j+1}:= \begin{cases}f_{j}(x) & \text { if } x \in \bar{\Omega} \backslash \bigcup_{i \in \mathbb{N}} \Omega_{i j} \\ h_{i j}(x) & \text { if } x \in \Omega_{i j} \text { for some } i \in \mathbb{N}\end{cases}
$$

By Lemma 5, it is homeomorphism and, moreover, properties 1) -4) hold for $j+1$. In addition,

(a) $\exists C>0$ depending only on $n$ such that $\left|D f_{j+1}-D f_{j}\right| \leq C 2^{-j}$ a.e. in $\bigcup_{i}\left(\Omega_{i j} \backslash \tilde{\Omega}_{i j}\right)$.

(b) $\left|\left\{x \in \bigcup_{i \in \mathbb{N}} \tilde{\Omega}_{i}:\left|D f_{j+1}(x)\right|>t\right\}\right| \lesssim 2^{-j}|\Omega| t^{-m}$. 
To get property [5) for $f_{j+1}$ we proceed as follows. Let

$$
t>\frac{1+2^{-1} C}{1+2^{-2}}
$$

On the one hand, thanks to (国) and (5),

$$
\begin{aligned}
\sum_{i=1}^{\infty}\left|\left\{x \in \Omega_{i j} \backslash \tilde{\Omega}_{i j}:\left|D f_{j+1}(x)\right|>t\right\}\right| & \leq\left|\left\{x \in \Omega:\left|D f_{j}(x)\right|>t-C 2^{-j}\right\}\right| \\
& \lesssim|\Omega|\left(t-C 2^{-j}\right)^{-m} \prod_{k=0}^{j-1}\left(1-2^{-k-1}\right)^{-m}\left(1+2^{-k}\right),
\end{aligned}
$$

but $\left(t-C 2^{-j}\right)^{-m} \leq\left(1-2^{-j-1}\right)^{-m} t^{-m}$, hence

$$
\sum_{i=1}^{\infty}\left|\left\{x \in \Omega_{i j} \backslash \tilde{\Omega}_{i j}:\left|D f_{j+1}(x)\right|>t\right\}\right| \lesssim|\Omega|\left(1-2^{-j-1}\right)^{-m} t^{-m} \prod_{k=0}^{j-1}\left(1-2^{-k-1}\right)^{-m}\left(1+2^{-k}\right) .
$$

Summing this estimate with that of (b) we obtain

$$
\begin{aligned}
\left|\left\{x \in \Omega:\left|D f_{j+1}(x)\right|>t\right\}\right| & \lesssim|\Omega| t^{-m}\left[\left(1-2^{-j-1}\right)^{-m} \prod_{k=0}^{j-1}\left(1-2^{-k-1}\right)^{-m}\left(1+2^{-k}\right)+2^{-j}\right] \\
& \leq|\Omega| t^{-m} \prod_{k=0}^{j}\left(1-2^{-k-1}\right)^{-m}\left(1+2^{-k}\right),
\end{aligned}
$$

so property [5) holds for $j+1$. This concludes the construction of $\left\{f_{j}\right\}_{j \in \mathbb{N}}$ with properties 1) (5).

As in the proof of Lemma 7, property 2) implies that the sequence $\left\{f_{j}\right\}_{j=1}^{\infty}$ converges in $C^{\alpha}$ to a function $f$ that is a homeomorphism with a $C^{\alpha}$ inverse. It also shows property $\left.⿴\right)$ of the statement. Property 4$)$, on the other hand, shows that $\left\{D f_{j}\right\}_{j \in \mathbb{N}}$ converges in $L^{1}$. Consequently, $f \in W^{1,1}\left(\Omega, \mathbb{R}^{n}\right)$.

Now, for a subsequence $D f_{j} \rightarrow D f$ a.e., so, thanks to the continuity of the singular values (see, e.g., (23)), we obtain from property 3 ) that $D f(x) \in \Gamma_{+}$and $\operatorname{rank}(D f(x))<m$ a.e. in $\Omega$. From the convergence $D f_{j} \rightarrow D f$ in measure and property 5 ) we have

$$
\frac{|\{x \in \Omega:|D f(x)|>t\}|}{|\Omega|} \lesssim t^{-m} \prod_{k=0}^{\infty}\left(1-2^{-k-1}\right)^{-m}\left(1+2^{-k}\right) \lesssim t^{-m},
$$

and therefore, $D f \in L^{m, w}(\Omega)$.

Finally, to prove that $f$ is the gradient of a convex function, we apply a standard argument. We assume, without loss of generality, that $\Omega$ is connected; otherwise, we would argue with each connected component. Take a ball $B$ containing $\bar{\Omega}$ and define $\tilde{f}: B \rightarrow \mathbb{R}^{n}$ as $f$ in $\Omega$ and the identity outside $\Omega$. Then $\tilde{f} \in W^{1,1}\left(B, \mathbb{R}^{n}\right)$. Choose a family $\left\{\eta_{\varepsilon}\right\}_{\varepsilon>0}$ of standard mollifiers, 
and define $f_{\varepsilon}:=\tilde{f} * \eta_{\varepsilon}$ in a ball $B_{\varepsilon} \subset B$ containing $\bar{\Omega}$. Then, $D f_{\varepsilon}(x) \in \Gamma_{+}$for all $x \in B_{\varepsilon}$. Consequently, the differential 1-form $\alpha_{\varepsilon}:=\sum_{i=1}^{n} f_{\varepsilon}^{i} d x_{i}$ defined in $B_{\varepsilon}$ is closed, i.e., $d \alpha_{\varepsilon}=0$, thanks to the symmetry of $D f_{\varepsilon}$. Here $f_{\varepsilon}^{i}$ are the components of $f_{\varepsilon}$. By Poincaré's lemma, $\alpha_{\varepsilon}$ is exact, i.e., there exists a smooth function $u_{\varepsilon}: B_{\varepsilon} \rightarrow \mathbb{R}$ such that $d u_{\varepsilon}=\alpha_{\varepsilon}$, so $\nabla u_{\varepsilon}=f_{\varepsilon}$. We can take $u_{\varepsilon}$ such that $\int_{\Omega} u_{\varepsilon}=0$. As the Hessian of $u_{\varepsilon}$ is symmetric positive semidefinite, $u_{\varepsilon}$ is convex. Now, $f_{\varepsilon} \rightarrow f$ in $W^{1,1}\left(\Omega, \mathbb{R}^{n}\right)$ as $\varepsilon \rightarrow 0$. Thanks to the Poincaré inequality, there exists $u \in W^{2,1}(\Omega)$ such that $u_{\varepsilon} \rightarrow u$ in $W^{2,1}(\Omega)$. Therefore, $\nabla u=f$. Moreover, $u$ is convex as a limit of convex functions. Theorem 1 is proved.

In fact, since in our construction the sets $E_{j}$ approximate planes of dimension $m-1$, our function satisfies rank $(D f(x))=m-1$ a.e. in $\Omega$. One can also see that, in our construction, $f \notin W^{1, m}\left(B, \mathbb{R}^{n}\right)$ for any open $B \subset \Omega$.

\section{Sharpness of the result}

Theorem 1 is sharp in the following sense.

Theorem 12. Let $\Omega \subset \mathbb{R}^{n}$ be open and bounded, and $m \in\{2, \ldots, n\}$. Let $f: \bar{\Omega} \rightarrow \mathbb{R}^{n}$ be a homeomorphism in $W^{1, p}\left(\Omega, \mathbb{R}^{n}\right)$ such that $\left.f\right|_{\partial \Omega}=\left.\mathrm{id}\right|_{\partial \Omega}$. Assume one of the following:

a) $p=m$ and $f$ is Hölder continuous.

b) $p>m$.

Then $\operatorname{rank}(D f(x)) \geq m$ for all $x$ in a subset of $\Omega$ of positive measure.

To prove this theorem we need to show the validity of the area formula for restrictions to planes of dimension $m$. Given $f \in W^{1, p}\left(\Omega, \mathbb{R}^{n}\right)$, we define $J_{m} f(x)=\sqrt{\operatorname{det} D f(x)^{T} D f(x)}$ for a.e. $x \in \Omega$. We denote by $\mathcal{H}^{m}$ the $m$-dimensional Hausdorff measure in $\mathbb{R}^{n}$; when $\mathcal{H}^{m}$ acts on subsets of coordinate planes of dimension $m$, it can be identified with the Lebesgue measure in $\mathbb{R}^{m}$.

Lemma 13. Let $f: \bar{\Omega} \rightarrow \mathbb{R}^{n}$ be injective and in $W^{1, p}\left(\Omega, \mathbb{R}^{n}\right)$. Assume that one of the alternatives $\square$ (b) of Theorem 12 holds. Then for almost every $y \in \mathbb{R}^{n-m}$ and all $\mathcal{H}^{m}$ measurable sets $E \subset \Omega \cap\left(\mathbb{R}^{m} \times\{y\}\right)$,

$$
\int_{E} J_{m} f d \mathcal{H}^{m}=\mathcal{H}^{m}(f(E)) .
$$

Proof. By Fubini's theorem, for a.e. $y \in \mathbb{R}^{n-m}$, the restriction $\left.f\right|_{\Omega \cap\left(\mathbb{R}^{m} \times\{y\}\right)}$ is in $W^{1, p}$ with respect to the $\mathcal{H}^{m}$ measure. Fix such a $y$. A standard approximation theorem (see, e.g., [14. Corollary 6.6.2]) shows that there exist sequences $\left\{f_{j}\right\}_{j \in \mathbb{N}}$ in $C^{1}\left(\mathbb{R}^{n}, \mathbb{R}^{n}\right)$ and $\left\{E_{j}\right\}_{j \in \mathbb{N}}$ of disjoint $\mathcal{H}^{m}$-measurable subsets of $E \cap\left(\mathbb{R}^{m} \times\{y\}\right)$ such that

$$
f(x)=f_{j}(x) \quad \text { and } \quad D f(x)=D f_{j}(x) \quad \text { for all } x \in E_{j} \text { and } j \in \mathbb{N}
$$


and $\mathcal{H}^{m}\left(E \backslash \bigcup_{j=1}^{\infty} E_{j}\right)=0$. Thus,

$$
\int_{E} J_{m} f d \mathcal{H}^{m}=\sum_{j=1}^{\infty} \int_{E_{j}} J_{m} f d \mathcal{H}^{m} .
$$

Now, for each $j \in \mathbb{N}$, thanks to the area formula for regular maps (see [17, Theorem 3.2.3]) and the fact that $f$ is injective,

$$
\begin{aligned}
& \int_{E_{j}} J_{m} f d \mathcal{H}^{m}=\int_{E_{j}} J_{m} f_{j} d \mathcal{H}^{m}=\int_{\mathbb{R}^{n}} \#\left\{E_{j} \cap f_{j}^{-1}(y)\right\} d \mathcal{H}^{m}(y) \\
& =\int_{\mathbb{R}^{n}} \#\left\{E_{j} \cap f^{-1}(y)\right\} d \mathcal{H}^{m}(y)=\mathcal{H}^{m}\left(f\left(E_{j}\right)\right) .
\end{aligned}
$$

Therefore,

$$
\int_{E} J_{m} f d \mathcal{H}^{m}=\mathcal{H}^{m}\left(f\left(\bigcup_{j=1}^{\infty} E_{j}\right)\right) .
$$

Under assumptions 回) or [b) of Theorem [12, $\left.f\right|_{\Omega \cap\left(\mathbb{R}^{m} \times\{y\}\right)}$ satisfies the $m$-dimensional Luzin (N) condition, i.e., given $A \subset \Omega \cap\left(\mathbb{R}^{m} \times\{y\}\right)$ such that $\mathcal{H}^{m}(A)=0$ then $\mathcal{H}^{m}(f(A))=0$. The proof under $\square$ ) is due to [24, Theorem 1.1] (with $\lambda=0$ in the notation there), while the proof under b) is classical [26]. In either case,

$$
\mathcal{H}^{m}\left(f\left(E \backslash \bigcup_{j=1}^{\infty} E_{j}\right)\right)=0
$$

and the proof is concluded.

Now we can prove Theorem 12 .

Proof of Theorem 12. Suppose, for a contradiction, that $\operatorname{rank}(D f(x))<m$ for a.e. $x \in \Omega$. Then $J_{m} f=0$ a.e., thanks to the Cauchy-Binet formula. Then, for a.e. $y \in \mathbb{R}^{m}$ we have $J_{m} f=0 \mathcal{H}^{m}$-a.e. in $\Omega \cap\left(\mathbb{R}^{m} \times\{y\}\right)$ and, by Lemma 13,

$$
\mathcal{H}^{m}\left(f\left(\Omega \cap\left(\mathbb{R}^{m} \times\{y\}\right)\right)\right)=0 .
$$

Let $P_{m}: \mathbb{R}^{n} \rightarrow \mathbb{R}^{m}$ be the projection over the first $m$ coordinates: $P_{m}\left(x_{1}, \ldots, x_{n}\right)=$ $\left(x_{1}, \ldots, x_{m}\right)$, and, for any $y \in \mathbb{R}^{n-m}$, define the set $\Omega_{y}=P_{m}\left(\Omega \cap\left(\mathbb{R}^{m} \times\{y\}\right)\right)$ and the function $g_{y}: \Omega_{y} \rightarrow \mathbb{R}^{m}$

$$
g_{y}(x)=P_{m}(f(x, y)) .
$$

Since $f=\mathrm{id}$ on $\partial \Omega$ and $\partial \Omega_{y}=P_{m}\left(\partial \Omega \cap\left(\mathbb{R}^{m} \times\{y\}\right)\right)$, we have that $g_{y}=\mathrm{id}$ on $\partial \Omega_{y}$. Using now degree theory, this implies

$$
\operatorname{deg}\left(g_{y}, \Omega_{y}, \cdot\right)=\operatorname{deg}\left(\mathrm{id}, \Omega_{y}, \cdot\right)
$$


and, consequently, $\Omega_{y} \subset g_{y}\left(\Omega_{y}\right)$ (see, e.g., [12, Theorem 3.1]). Fix $y \in \mathbb{R}^{n-m}$ such that (49) holds and $\Omega_{y} \neq \emptyset$. As $P_{m}$ is 1-Lipschitz and $\Omega_{y}$ is open, we find that

$$
0<\mathcal{H}^{m}\left(\Omega_{y}\right) \leq \mathcal{H}^{m}\left(g_{y}\left(\Omega_{y}\right)\right) \leq \mathcal{H}^{m}\left(f\left(\Omega \cap\left(\mathbb{R}^{m} \times\{y\}\right)\right)\right),
$$

which contradicts (49) and completes the proof.

As can be seen from the proof, in Theorem 12 and Lemma 13, conditions $\square$ ( $)$ b) can be replaced by any other assumption implying Luzin's condition $(\mathrm{N})$ in $\mathbb{R}^{m}$. As mentioned in the proof, the paper [24] shows some of those conditions.

\section{Acknowledgements}

The authors have been supported by Project MTM2014-57769-C3-1-P of the Spanish Ministry of Economy and Competitivity and the ERC Starting grant no. 307179. C.M.-C. has also been supported by the "Ramón y Cajal" programme and the European Social Fund.

\section{References}

[1] G. Alberti And L. Ambrosio, A geometrical approach to monotone functions in $\mathbb{R}^{n}$, Math. Z., 230 (1999), pp. 259-316.

[2] K. Astala, D. Faraco, and L. Székelyhidi, Jr., Convex integration and the $L^{p}$ theory of elliptic equations, Ann. Sc. Norm. Super. Pisa Cl. Sci. (5), 7 (2008), pp. 1-50.

[3] J. M. BALL, Global invertibility of Sobolev functions and the interpenetration of matter, Proc. Roy. Soc. Edinburgh Sect. A, 88 (1981), pp. 315-328.

[4] N. Boros, L. Székelyhidi, Jr., And A. Volberg, Laminates meet Burkholder functions, J. Math. Pures Appl. (9), 100 (2013), pp. 687-700.

[5] Y. Brenier, Polar factorization and monotone rearrangement of vector-valued functions, Comm. Pure Appl. Math., 44 (1991), pp. 375-417.

[6] R. ČERnÝ, Homeomorphism with zero Jacobian: sharp integrability of the derivative, J. Math. Anal. Appl., 373 (2011), pp. 161-174.

[7] — Bi-Sobolev homeomorphism with zero minors almost everywhere, Adv. Calc. Var., 8 (2015), pp. $1-30$.

[8] S. Conti, D. Faraco, And F. Maggi, A new approach to counterexamples to $L^{1}$ estimates: Korn's inequality, geometric rigidity, and regularity for gradients of separately convex functions, Arch. Ration. Mech. Anal., 175 (2005), pp. 287-300.

[9] S. Conti, D. Faraco, F. Maggi, and S. Müller, Rank-one convex functions on $2 \times 2$ symmetric matrices and laminates on rank-three lines, Calc. Var. Partial Differential Equations, 24 (2005), pp. 479-493.

[10] B. Dacorogna, Direct methods in the calculus of variations, vol. 78 of Applied Mathematical Sciences, Springer-Verlag, Berlin, 1989. 
[11] G. De Philippis And A. Figalli, The Monge-Ampère equation and its link to optimal transportation, Bull. Amer. Math. Soc. (N.S.), 51 (2014), pp. 527-580.

[12] K. Deimling, Nonlinear functional analysis, Springer, Berlin, 1985.

[13] L. D'Onofrio, S. Hencl, and R. Schiattarella, Bi-Sobolev homeomorphism with zero Jacobian almost everywhere, Calc. Var. Partial Differential Equations, 51 (2014), pp. 139-170.

[14] L. C. Evans And R. F. Gariepy, Measure theory and fine properties of functions, Studies in Advanced Mathematics, CRC Press, Boca Raton, FL, 1992.

[15] D. Faraco, Milton's conjecture on the regularity of solutions to isotropic equations, Ann. Inst. H. Poincaré Anal. Non Linéaire, 20 (2003), pp. 889-909.

[16] — Wild mappings built on unbounded laminates, in Proceedings of the Workshop "New Developments in the Calculus of Variations", M. Carozza, L. D'Onofrio, L. Greco, G. Moscariello, A. Passarelli di Napoli, and C. Sbordone, eds., vol. 2 of Sezione statistico-matematica, Università degli studi del Sannio, Edizioni Scientifiche Italiane, 2006, pp. 89-108.

[17] H. Federer, Geometric measure theory, Die Grundlehren der mathematischen Wissenschaften, Band 153, Springer-Verlag New York Inc., New York, 1969.

[18] I. Gohberg, S. Goldberg, And M. A. KaAshoek, Classes of linear operators. Vol. I, vol. 49 of Operator Theory: Advances and Applications, Birkhäuser Verlag, Basel, 1990.

[19] P. HajŁasz, Change of variables formula under minimal assumptions, Colloq. Math., 64 (1993), pp. $93-101$.

[20] S. Hencl, Sobolev homeomorphism with zero Jacobian almost everywhere, J. Math. Pures Appl. (9), 95 (2011), pp. 444-458.

[21] J. Kauhanen, P. Koskela, and J. Malý, Mappings of finite distortion: condition N, Michigan Math. J., 49 (2001), pp. 169-181.

[22] B. Kirchieim and J. Kristensen, Automatic convexity of rank-1 convex functions, C. R. Math. Acad. Sci. Paris, 349 (2011), pp. 407-409.

[23] — On rank one convex functions that are homogeneous of degree one, Arch. Ration. Mech. Anal., To appear (2016), pp. 1-32.

[24] P. Koskela, J. Malý, And T. ZÜrcher, Luzin's condition (N) and modulus of continuity, Adv. Calc. Var., 8 (2015), pp. 155-171.

[25] Z. Liu And J. MalÝ, A strictly convex Sobolev function with null Hessian minors. Preprint available at http://msekce.karlin.mff.cuni.cz/ms-preprints/kma-preprints/2015-pap/2015-499.pdf.

[26] M. Marcus and V. J. Mizel, Transformations by functions in Sobolev spaces and lower semicontinuity for parametric variational problems, Bull. Amer. Math. Soc., 79 (1973), pp. 790-795.

[27] S. MüLLER, Variational models for microstructure and phase transitions, in Calculus of variations and geometric evolution problems (Cetraro, 1996), vol. 1713 of Lecture Notes in Math., Springer, Berlin, 1999, pp. 85-210.

[28] S. MÜller AND V. ŠverÁK, Convex integration for Lipschitz mappings and counterexamples to regularity, Ann. of Math. (2), 157 (2003), pp. 715-742.

[29] P. Pedregal, Laminates and microstructure, European J. Appl. Math., 4 (1993), pp. 121-149.

[30] C. Villani, Topics in optimal transportation, vol. 58 of Graduate Studies in Mathematics, American Mathematical Society, Providence, RI, 2003. 